\title{
CYP1A1 Mspl and exon7 gene polymorphisms and lung cancer risk: An updated meta-analysis and review
}

\author{
Ping Zhan ${ }^{1+}$, Qin Wang ${ }^{2 \dagger}$, Qian Qian', Shu-Zhen Wei ${ }^{3}$ and Li-Ke Yu ${ }^{1 *}$
}

\begin{abstract}
Background: Many studies have examined the association between the CYP1A1 Mspl and exon 7 gene polymorphisms and lung cancer risk in various populations, but their results have been inconsistent.

Methods: To assess this relationship more precisely, a meta-analysis and review were performed. The PubMed, Embase, Web of Science, and CNKI database was searched for case-control studies published up to June 2010. Data were extracted and pooled odds ratios (OR) with 95\% confidence intervals (Cl) were calculated.

Results: Ultimately, 64 studies, comprising 18,397 subjects from 49 case-control studies of the Mspl genotype and 18,518 patients from 40 case-control studies of the exon 7 genotype, were included. A significantly elevated lung cancer risk was associated with $2 \mathrm{Mspl}$ genotype variants (for type $C$ vs Type $\mathrm{A}: \mathrm{OR}=1.26,95 \% \mathrm{Cl}=1.12-1.42$; for types $\mathrm{B}$ and $\mathrm{C}$ combined vs Type A: $\mathrm{OR}=1.20,95 \% \mathrm{Cl}=1.13-1.28)$ in overall population. In the stratified analysis, a significant association was found in Asians, Caucasians, lung SCC, lung AC and Male population, not in mixed population, lung SCLC and Female population. However, inconsistent results were observed for CYP1A1 exon7 in our meta-analysis, two variants of the exon 7 polymorphism were associated with a significantly higher risk for lung cancer (for Val $\mathrm{Nal}$ vs lle/lle: $\mathrm{OR}=1.24,95 \% \mathrm{Cl}=1.09-1.42$; for (lle/ $\mathrm{Val}+\mathrm{Val} / \mathrm{Val}$ ) vs lle/lle: $\mathrm{OR}=1.15,95 \% \mathrm{Cl}=1.07-1.24$ ) in overall population. In the stratified analysis, a significant assocation was found in Asians, Caucasians, lung SCC and Female population, not in mixed population, lung AD, lung SCLC and Male population. Additionally, a significant association was found in smoker population and not found in non-smoker populations for CYP1A1 Mspl and exon7 gene.
\end{abstract}

Conclusions: This meta-analysis suggests that the Mspl and exon 7 polymorphisms of CYP1A1 correlate with increased lung cancer susceptibility and there is an interaction between two genotypes of CYP1A1 polymorphism and smoking, but these associations vary in different ethnic populations, histological types of lung caner and gender of case and control population.

Keywords: CYP1A1, Polymorphism, Lung cancer, Susceptibility, Meta-analysis

\section{Introduction}

Lung cancer remains the most lethal cancer worldwide, despite improvements in diagnostic and therapeutic techniques [1]. Its incidence has not peaked in many parts of world, particularly in China, which has become a major public health challenge all the world [2]. The mechanism of lung carcinogenesis is not understood. Although

\footnotetext{
*Correspondence: yulike_nanjing@163.com

† Contributed equally

'First Department of Respiratory Medicine, Nanjing Chest Hospital, 215

Guangzhou Road, Nanjing 210029, China

Full list of author information is available at the end of the article
}

cigarette smoking is the major cause of lung cancer, not all smokers develop lung cancer [3], which suggests that other causes such as genetic susceptibility might contribute to the variation in individual lung cancer risk $[4,5]$. Many environmental carcinogens require metabolic activation by drug-metabolizing enzymes. In recent years, several common low-penetrance genes have been implicated as potential lung cancer susceptibility genes.

Cytochrome P450 1A1 (CYP1A1) metabolizes several suspected procarcinogens, particularly polycyclic aromatic hydrocarbons (PAHs), into highly reactive intermediates [6]. These compounds bind to DNA to form adducts,

\section{Ciomed Central}


which, if unrepaired, can initiate or accelerate carcinogenesis. Although PAHs are ubiquitous in the environment, notable sources of exposure that cause the greatest concern include smoking, air pollution, diet, and certain occupations [7]. Two functionally important nonsynonymous polymorphisms have been described for the CYP1A1 gene, a base substitution at codon 462 in exon 7, resulting in substitution of isoleucine with valine (Ile462 Val (exon 7)) (National Center for Biotechnology Information single nucleotide polymorphism(SNP) identifier rs1048943; adenine (A) to guanine (G) substitution at nucleotide 2455 (2455A.G)) and a point mutation (thymine (T) to cytosine (C)) at the MspI site in the 3'-untranslated region (rs4646903;3801T.C) [8]. The MspI restriction site polymorphism resulted in three genotypes: a predominant homozygous $\mathrm{m} 1$ allele without the MspI site (genotype A), the heterozygote (genotype $\mathrm{B}$ ), and a homozygous rare $\mathrm{m} 2$ allele with the MspI site (genotype C). The exon 7 restriction site polymorphism resulted in three genotypes: a predominant homozygous (Ile/lle), the heterozygote (Ile/Val), and the rare homozygous(Val/Val).

An association between CYP1A1 polymorphisms and lung cancer was first reported by Kawajiri and co-workers in 1990 among an Asian study population (Febs Lett 1990;263:131-133)[9], after which many studies analyzed the influence of CYP1A1 polymorphisms on lung cancer risk; no clear consensus, however, was reached. Moreover, 3 meta-analyses have reported conflicting results. Houlston RS [10] found no statistically significant association between the MspI polymorphism and lung cancer risk in 2000, in a meta-analysis performed by Le Marchand L et al. [11] included only 11 studies, the exon 7 polymorphism did not correlate with lung cancer risk. Shi $\times$ [12], however, noted a greater risk of lung cancer for CYP1A1 MspI and exon 7 polymorphism carriers in a meta-analysis that included only Chinese population.

A single study might not be powered sufficiently to detect a small effect of the polymorphisms on lung cancer, particularly in relatively small sample sizes. Various types of study populations and study designs might also have contributed to these disparate findings. To clarify the effect of the CYP1A1 polymorphism on the risk for lung cancer, we performed an updated meta-analysis of all eligible case-control studies to date and conducted the subgroup analysis by stratification according to the ethnicity source, histological types of lung caner, gender and smoking status of case and control population.

\section{Materials and methods}

\subsection{Publication search}

We searched for studies in the PubMed, Embase, Web of Science, and CNKI (China National Knowledge Infrastructure) electronic databases to include in this meta-analysis, using the terms "CYP1A1," "Cytochrome P450 1A1," "polymorphism," and "lung cancer." An upper date limit of June, 2010 was applied; no lower date limit was used. The search was performed without any restrictions on language and was focused on studies that had been conducted in humans. We also reviewed the Cochrane Library for relevant articles. Concurrently, the reference lists of reviews and retrieved articles were searched manually. When the same patient population appeared in several publications, only the most recent or complete study was included in this meta-analysis.

\subsection{Inclusion criteria}

For inclusion, the studies must have met the following criteria: they (1) evaluated CYP1A1 gene polymorphisms and lung cancer risk; (2) were case-control studies or nested-case control study; (3) supplied the number of individual genotypes for the CYP1A1 MspI and exon 7 polymorphisms in lung cancer cases and controls, respectively; and (4) demonstrated that the distribution of genotypes among controls were in Hardy-Weinberg equilibrium.

\subsection{Data extraction}

Information was extracted carefully from all eligible publications independently by 2 authors, based on the inclusion criteria above. Disagreements were resolved through a discussion between the 2 authors.

The following data were collected from each study: first author's surname, year of publication, ethnicity, total numbers of cases and controls, and numbers of cases and controls who harbored the MspI and exon 7 genotypes, respectively. If data from any category were not reported in the primary study, the items were designated "not applicable." We did not contact the author of the primary study to request the information. Ethnicities were categorized as Asian, Caucasian, and mixed. Histological type of lung cancer was divided to lung squamous carcinoma (SCC), adenocarcinoma (AC) and small cell lung cancer (SCLC) in our meta-analysis. The definition of smoking history is very complicated. The smoking histories covered different periods if changes in the number of cigarettes smoked per day or type of tobacco products occurred. Cigarette types were classified as filtered or unfiltered commercial products and local traditional hand-made khii yo and yamuan, both unfiltered. According to the general standards, non-smokers were defined as subjects who had smoked less than 100 cigarettes in their lifetime. Although the precise definition of neversmoking status varied slightly among the studies, the smoking status was classified as non-smokers (or never smoker) and smokers (regardless of the extent of smoking) in our meta-analysis. We did not require a minimum number of patients for a study to be included in our meta-analysis. 


\subsection{Statistical analysis}

OR (odds ratios) with 95\% CIs were used to determine the strength of association between the CYP1A1MspI and exon7 polymorphisms and lung cancer risk. We evaluated this risk with regard to combinations of variants (i.e., type B and type C for MspI and Ile/Val and $\mathrm{Val} / \mathrm{Val}$ for exon 7) versus the wild-type homozygotes (type A for MspI and Ile/Ile for exon 7).

The pooled ORs for the risk were calculated. Subgroup analyses were performed by ethnicity. Heterogeneity assumptions were assessed by chi-square-based Q-test [13]. A P value greater than 0.10 for the Q-test indicated a lack of heterogeneity among studies, so that the pooled OR estimate of each study was calculated by the fixedeffects model (the Mantel-Haenszel method) [14]. Otherwise, the random-effects model (the DerSimonian and Laird method) was used [15]. In addition, subgroup analysis stratified by ethnicity, gender and histological types of lung caner was also performed.

One-way sensitivity analyses were performed to determine the stability of the results-each individual study in the meta-analysis was omitted to reflect the influence of the individual dataset on the pooled OR [16].

Potential publication biases were estimated by funnel plot, in which the standard error of log (OR) of each study was plotted against its log (OR). An asymmetrical plot suggests a publication bias. Funnel plot asymmetry was assessed by Egger's linear regression test, a linear regression approach that measures the funnel plot asymmetry on a natural logarithm scale of the OR. The significance of the intercept was determined by t-test, as suggested by Egger $(\mathrm{P}<0.05$ was considered a statistically significant publication bias) [17].

All calculations were performed using STATA, version 10.0 (Stata Corporation, College Station, TX).

\section{Results}

\subsection{Study characteristics}

Two hundred and fifty-seven potentially relevant citations were reviewed, and 64 publications met the inclusion criteria and included in our meta-analysis [9,18-80]. Study search process was shown in Figure 1. Table 1 presents the principal characteristics of these studies. For the MspI genotype, 49 studies of 7658 lung cancer cases and 11839 controls were ultimately analyzed. Raimondi's study [58] sorted the data for Caucasians and Asians; therefore, each group in the study was considered separately in the pooled subgroup analyses. For the exon7 polymorphism, 40 studies of 6067 lung cancer cases and 12451 controls were analyzed.

Of the 64 publications, 50 were published in English and 14 were written in Chinese. The sample sizes ranged from 104 to 1824 . All cases were histologically confirmed. The controls were primarily healthy populations and matched for age, ethnicity, and smoking status.

There were 26 groups of Asians, 11 groups of Caucasians, and 12 mixed populations for MspI; for exon7, there were 22 groups of Asians, 10 groups of Caucasians, and 8 mixed populations. All polymorphisms in the control subjects were in Hardy-Weinberg equilibrium.

\subsection{Meta-analysis results}

\subsubsection{Association of CYP1A1 Mspl variant with lung cancer} risk

Table 2 lists the primary results. Overall, a significantly elevated risk of lung cancer was associated with 2 variants of CYP1A1 MspI (for Type C vs Type A: OR = 1.26, 95\% $\mathrm{CI}=1.12-1.42, P=0.003$ for heterogeneity; for types $\mathrm{B}$ and $C$ combined vs Type A: $\mathrm{OR}=1.20,95 \% \mathrm{CI}=1.13$ $1.28, P=0.000$ for heterogeneity) (Figure 2).

In the stratified analysis by ethnicity, significantly increased risks were observed among Asians for both type $C$ vs Type $A(O R=1.24,95 \% C I=1.12-1.43 ; P=0.004$ for heterogeneity), types $\mathrm{B}$ and $\mathrm{C}$ combined vs Type $\mathrm{A}(\mathrm{OR}=$ $1.30,95 \% \mathrm{CI}=1.17-1.44 ; P=0.002$ for heterogeneity). In Caucasians, there was also significant association in Type $\mathrm{C}$ vs Type A $(\mathrm{OR}=1.25 ; 95 \% \mathrm{CI}=1.09-1.36 ; P=0.052$ for heterogeneity), types $\mathrm{B}$ and $\mathrm{C}$ combined vs Type $\mathrm{A}(\mathrm{OR}=$ $1.35 ; 95 \% \mathrm{CI}=1.18-1.54 ; P=0.046$ for heterogeneity). However, in mixed populations, no significant associations were observed (Table 2).

Fourteen [9,19,22,24,26,29,31,32,40,47,53,58,64,78] out of 64 studies examined the association of CYP1A1 MspI genotype and the risk of different histological types of lung cancer including SCC, AC and SCLC. Among lung SCC and lung $A C$, significantly increased risks were observed for both type $C$ vs Type A, types B and $C$ combined vs Type A. However, among lung SCLC, no significant associations were observed for both type $C$ vs Type $A$ (OR = $0.96 ; 95 \% \mathrm{CI}=0.70-1.26 ; P=0.864$ for heterogeneity) or types $\mathrm{B}$ and $\mathrm{C}$ combined vs Type $\mathrm{A}(\mathrm{OR}=1.06 ; 95 \% \mathrm{CI}=$ 0.77-1.45; $P=0.976$ for heterogeneity) (Figure 3 ).

Seven $[45,56,61,64,74-76]$ out of 64 studies included the association of CYP1A1 MspI genotype and lung caner risk stratified by gender (Male and Female). For Male population (3 studies), significantly increased risks were observed for both type $C$ vs Type A (OR = 1.39; $95 \% \mathrm{CI}=1.23-1.79 ; P=0.210$ for heterogeneity), types $\mathrm{B}$ and $\mathrm{C}$ combined vs Type A $(\mathrm{OR}=1.46 ; 95 \% \mathrm{CI}=1.07$ 1.98; $P=0.380$ for heterogeneity). However, for Female population (7 studies), no significant associations were observed for both type $C$ vs Type A (OR $=0.92 ; 95 \%$ $\mathrm{CI}=0.84-1.16 ; P=0.003$ for heterogeneity) or types $\mathrm{B}$ and $\mathrm{C}$ combined vs Type $\mathrm{A}(\mathrm{OR}=0.85 ; 95 \% \mathrm{CI}=0.71$ $1.02 ; P=0.000$ for heterogeneity) (Figure 4 ). 


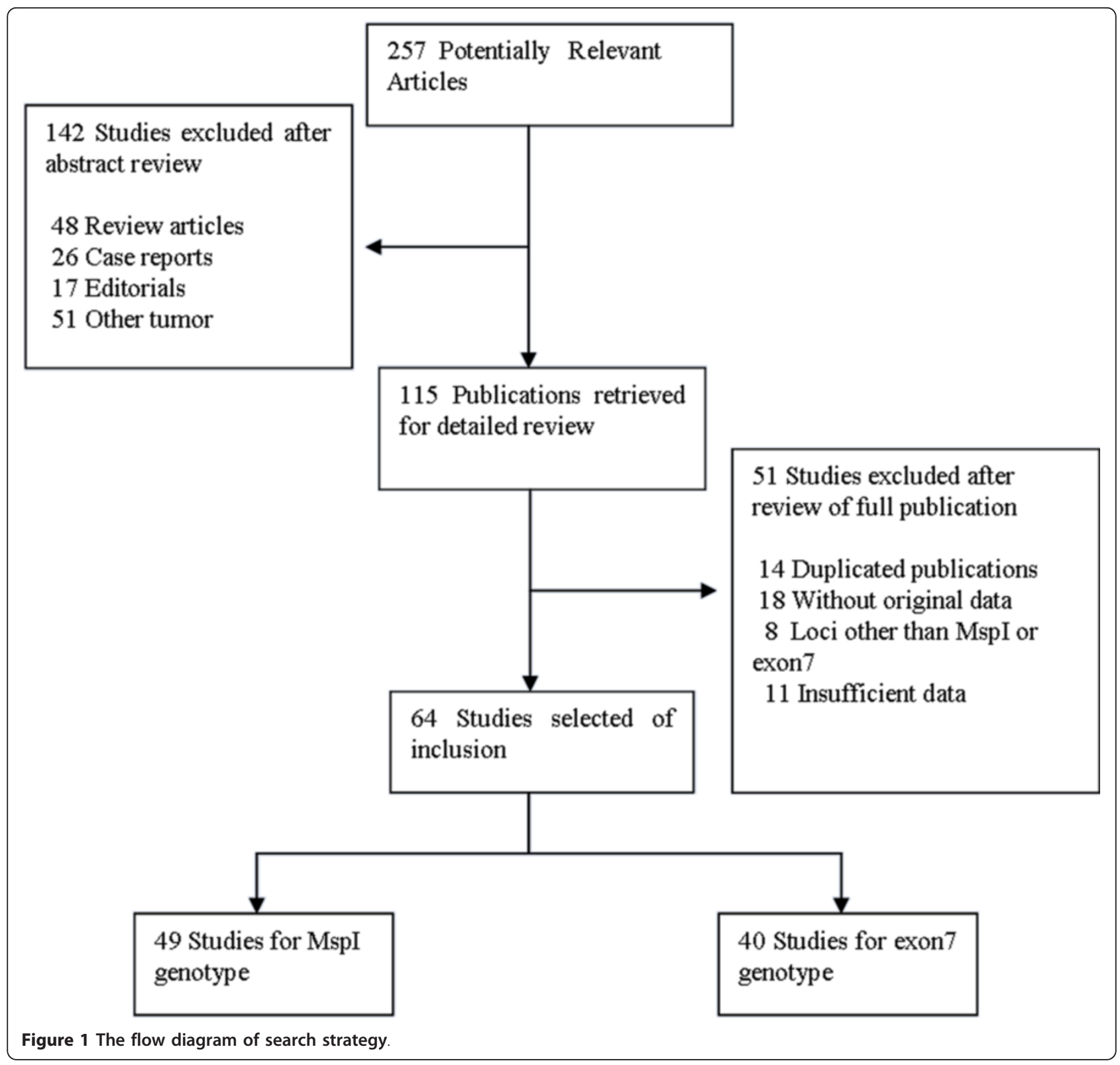

Thirteen $[24,31,47,56,59-61,64,72,75,78]$ out of 64 studies included the association of CYP1A1 MspI genotype and lung caner risk stratified by smoking status (nonsmokers or never smokers and smokers). For smokers, significantly increased risks were observed for both type $\mathrm{C}$ vs Type $\mathrm{A}(\mathrm{OR}=1.62 ; 95 \% \mathrm{CI}=1.33-1.96 ; P=0.000$ for heterogeneity), types $\mathrm{B}$ and $\mathrm{C}$ combined vs Type A $(\mathrm{OR}=1.75 ; 95 \% \mathrm{CI}=1.44-2.13 ; P=0.003$ for heterogeneity). However, for non-smokers, no significant associations were observed for both type $C$ vs Type $A(O R=$ $1.18 ; 95 \% \mathrm{CI}=0.96-1.186 ; P=0.086$ for heterogeneity) or types B and C combined vs Type A (OR = 1.09; 95\% $\mathrm{CI}=0.90-1.33 ; P=0.114$ for heterogeneity) (Figure 5 ).
3.2.2 Association of CYP1A1 exon7 variant with lung cancer risk

For all studies in the meta-analysis, the genotype, an increased risk for lung cancer was associated with 2 exon7 variants (for Val/Val vs Ile/Ile: $\mathrm{OR}=1.24,95 \% \mathrm{CI}=1.09$ $1.42, P=0.004$ for heterogeneity; for Ile/Val and Val/Val combined vs Ile/Ile: $\mathrm{OR}=1.15,95 \% \mathrm{CI}=1.07-1.24, P=$ 0.000 for heterogeneity) (Figure 6).

In the stratified analysis by ethnicity, the risk was higher in Asian carriers of $\mathrm{Val} / \mathrm{Val}$ vs Ile/Ile $(\mathrm{OR}=1.22,95 \%$ $\mathrm{CI}=1.16-1.59 ; P=0.016$ for heterogeneity), Ile/Val and $\mathrm{Val} / \mathrm{Val}$ combined vs Ile/Ile $(\mathrm{OR}=1.21,95 \% \mathrm{CI}=1.09$ $1.34 ; P=0.000$ for heterogeneity). A significant association 
Table 1 Distribution of CYP1A1 Mspl and exon7 genotypes among lung cancer cases and controls included in this meta-analysis

\begin{tabular}{|c|c|c|c|c|c|c|c|c|c|c|c|c|c|c|}
\hline \multirow[t]{2}{*}{ First author-year } & \multirow[t]{2}{*}{ Ethnicity(country of origin) } & \multirow[t]{2}{*}{$\begin{array}{l}\text { Total sample size } \\
\text { (case/control) }\end{array}$} & \multicolumn{3}{|c|}{$\begin{array}{l}\text { Lung cancer cases } \\
\text { of Mspl genotype }\end{array}$} & \multicolumn{3}{|c|}{$\begin{array}{l}\text { Controls of } \\
\text { Mspl genotype }\end{array}$} & \multicolumn{3}{|c|}{$\begin{array}{l}\text { Lung cancer cases } \\
\text { of exon7 genotype }\end{array}$} & \multicolumn{3}{|c|}{ Controls of exon7 genotype } \\
\hline & & & Type B & Type C & Type A & Type B & Type C & Type A & Ile/Val & Val/Val & Ile/lle & Ile/Val & Val/Val & Ile/lle \\
\hline Kawajiri K-1990 & Asia(Japan) & $68 / 104$ & 28 & 16 & 24 & 42 & 11 & 51 & NA & NA & NA & NA & NA & NA \\
\hline Tefre T-1991 & Caucasian(Norway) & $221 / 212$ & 47 & 2 & 172 & 43 & 2 & 167 & NA & NA & NA & NA & NA & NA \\
\hline Hirvonen A-1992 & Caucasian(Finnish) & $87 / 121$ & 22 & 0 & 65 & 24 & 2 & 95 & NA & NA & NA & NA & NA & NA \\
\hline Shields PG-1993 & Mixed populations & $56 / 48$ & 11 & 2 & 43 & 12 & 3 & 33 & NA & NA & NA & NA & NA & NA \\
\hline Nakachi K-1993 & Asia(Japan) & $31 / 127$ & 7 & 13 & 11 & 55 & 11 & 61 & 11 & 6 & 14 & 44 & 4 & 79 \\
\hline Alexandrie AK-1994 & Caucasian(Sweden) & $296 / 329$ & 44 & 4 & 248 & 52 & 1 & 276 & 16 & 0 & 280 & 23 & 0 & 306 \\
\hline Kelsey K.T -1994 & Mixed(African Americans) & $72 / 97$ & 11 & 1 & 60 & 21 & 2 & 74 & NA & NA & NA & NA & NA & NA \\
\hline Cantlay AM-1995 & Caucasian(Edinburgh) & $129 / 281$ & NA & NA & NA & NA & NA & NA & 21 & 2 & 106 & 33 & 3 & 245 \\
\hline Kihara M-1995 & Asia(Japan) & $97 / 258$ & 45 & 16 & 36 & 105 & 41 & 112 & 31 & 5 & 59 & 98 & 14 & 143 \\
\hline Xu XP-1996 & Caucasian(USA) & $207 / 238$ & 35 & 2 & 170 & 48 & 2 & 233 & NA & NA & NA & NA & NA & NA \\
\hline Garcia-ClosaM-1997 & Mixed populations & $416 / 446$ & 75 & 4 & 337 & 73 & 4 & 369 & NA & NA & NA & NA & NA & NA \\
\hline Ishibe N-1997 & Mixed(Mexican and African) & $171 / 295$ & 68 & 12 & 91 & 106 & 35 & 154 & 31 & 7 & 132 & 70 & 20 & 204 \\
\hline Hong YS-1998 & Asia(Korean) & $85 / 63$ & 45 & 6 & 34 & 31 & 3 & 29 & 68 & 1 & 16 & 60 & 1 & 2 \\
\hline Taioli E-1998 & Mixed populations & $105 / 307$ & 30 & 9 & 59 & 101 & 18 & 170 & 8 & 1 & 94 & 18 & 0 & 272 \\
\hline Sugimura H-1998 & Asia(Japan) & $247 / 185$ & NA & NA & NA & NA & NA & NA & 94 & 28 & 125 & 84 & 7 & 94 \\
\hline Le Marchand L-1998 & Mixed populations & $341 / 456$ & 121 & 35 & 183 & 160 & 44 & 250 & 68 & 6 & 263 & 105 & 13 & 335 \\
\hline Xue KX-1999 & Asia(china) & 103/131 & NA & NA & NA & NA & NA & NA & 31 & 18 & 54 & 36 & 11 & 36 \\
\hline Hu YL-1999 & Asia(china) & $59 / 132$ & 22 & 15 & 22 & 76 & 22 & 34 & 33 & 7 & 19 & 102 & 9 & 21 \\
\hline London SJ-2000 & Asia(China) & $214 / 669$ & NA & NA & NA & NA & NA & NA & 39 & 8 & 167 & 130 & 27 & 512 \\
\hline Dresler CM-2000 & Caucasian(USA) & $158 / 149$ & & $37^{*}$ & 121 & & $17^{*}$ & 132 & NA & NA & NA & NA & NA & NA \\
\hline Song N-2001 & Asia(China) & $217 / 404$ & 129 & 28 & 60 & 175 & 56 & 173 & 130 & 9 & 78 & 181 & 13 & 210 \\
\hline Ratnasinghe D-2001 & Caucasian(USA) & $282 / 324$ & NA & NA & NA & NA & NA & NA & 36 & 3 & 243 & 48 & 3 & 273 \\
\hline Quinones L-2001 & Caucasians(Chile) & $60 / 174$ & 29 & 10 & 16 & 38 & 16 & 86 & 35 & 10 & 15 & 52 & 14 & 54 \\
\hline Chen S-2001 & Asia(china) & 106/106 & NA & NA & NA & NA & NA & NA & 38 & 10 & 58 & 33 & 3 & 70 \\
\hline Xue KX-2001 & Asia(china) & 106/106 & NA & NA & NA & NA & NA & NA & 38 & 10 & 58 & 33 & 3 & 33 \\
\hline Yin LH-2002 & Asia(china) & $84 / 84$ & 34 & 13 & 37 & 38 & 18 & 28 & NA & NA & NA & NA & NA & NA \\
\hline Zhou XW-2002 & Asia(china) & $92 / 98$ & 43 & 15 & 34 & 34 & 13 & 51 & 66 & 11 & 15 & 65 & 6 & 65 \\
\hline Cai XL-2003 & Asia(china) & $91 / 138$ & 23 & 36 & 32 & 46 & 39 & 53 & NA & NA & NA & NA & NA & NA \\
\hline Kiyohara C-2003 & Asia(Japan) & $158 / 259$ & 64 & 17 & 77 & 115 & 28 & 116 & NA & NA & NA & NA & NA & NA \\
\hline Taioli E-2003 & Mixed populations & $\begin{array}{l}\text { 109/424 Mspl } \\
\text { 110/707exon7 }\end{array}$ & 20 & 5 & 84 & 75 & 4 & 345 & 16 & 1 & 93 & 70 & 2 & 635 \\
\hline Wang J-2003 & Asia(china) & $162 / 181$ & 76 & 22 & 64 & 78 & 38 & 65 & NA & NA & NA & NA & NA & NA \\
\hline Dialyna IA-2003 & Caucasians (Greek) & $122 / 178$ & 28 & 5 & 89 & 45 & 3 & 130 & NA & NA & NA & NA & NA & NA \\
\hline Dong CT-2004 & Asia(china) & $82 / 91$ & NA & NA & NA & NA & NA & NA & 36 & 18 & 28 & 32 & 10 & 32 \\
\hline Gu YF-2004 & Asia(china) & $180 / 224$ & & $129 *$ & 51 & & $138^{*}$ & 86 & NA & NA & NA & NA & NA & NA \\
\hline Liang GY-2004 & Asia(china) & 152/152 & 82 & 20 & 50 & 71 & 11 & 70 & NA & NA & NA & NA & NA & NA \\
\hline
\end{tabular}


Table 1 Distribution of CYP1A1 Mspl and exon7 genotypes among lung cancer cases and controls included in this meta-analysis (Continued)

\begin{tabular}{|c|c|c|c|c|c|c|c|c|c|c|c|c|c|c|}
\hline Chen SD-2004 & Asia(china) & $58 / 62$ & 15 & 23 & 20 & 20 & 18 & 24 & NA & NA & NA & NA & NA & NA \\
\hline Yang XR-2004 & Asia(China) & 200/144 & NA & NA & NA & NA & NA & NA & 96 & 11 & 90 & 39 & 7 & 98 \\
\hline Sobti RC-2004 & Asia(India) & $100 / 76$ & 45 & 6 & 49 & 29 & 5 & 42 & 67 & 29 & 4 & 53 & 15 & 8 \\
\hline Wenzlaff AS-2005 & Caucasian(USA) & $128 / 181$ & 35 & 0 & 93 & 30 & 4 & 116 & $5^{\#}$ & & 124 & $14^{\#}$ & & 134 \\
\hline Wrensch MR-2005 & Mixed populations & 371/944 Mspl 363/930exon7 & & $166^{*}$ & 205 & & $472^{*}$ & 472 & & $64^{\#}$ & 302 & & $219^{\#}$ & 711 \\
\hline Ng DP-2005 & Asia(Singapore) & $126 / 162$ & 61 & 22 & 41 & 87 & 19 & 56 & 39 & 13 & 74 & 63 & 7 & 91 \\
\hline Larsen EJ-2005 & Caucasians(Australia) & $1050 / 581$ & NA & NA & NA & NA & NA & NA & 84 & 8 & 958 & 27 & 2 & 552 \\
\hline Raimondi S-2005 & Caucasians & $\begin{array}{l}\text { 165/519 Mspl } \\
\text { 175/723exon7 }\end{array}$ & & $43^{*}$ & 122 & & $102^{*}$ & 417 & & $32^{\#}$ & 143 & & $67^{\#}$ & 656 \\
\hline Raimondi S-2005-2 & Asians & $\begin{array}{l}\text { 46/138 Mspl } \\
60 / 212 \text { exon7 }\end{array}$ & & $28^{*}$ & 18 & & $95^{*}$ & 43 & & $30^{\#}$ & 30 & & $96^{\#}$ & 116 \\
\hline Sreeja L-2005 & Asia(Indian) & $146 / 146$ & 53 & 22 & 71 & 45 & 8 & 93 & NA & NA & NA & NA & NA & NA \\
\hline Adonis M-2005 & Mixed populations & $57 / 103$ & 31 & 11 & 15 & 33 & 26 & 44 & NA & NA & NA & NA & NA & NA \\
\hline Belogubova-2006 & Caucasians (Russian) & $141 / 450$ & 35 & 2 & 104 & 90 & 3 & 357 & NA & NA & NA & NA & NA & NA \\
\hline Li DR-2006 & Asia(china) & $150 / 152$ & NA & NA & NA & NA & NA & NA & 104 & 14 & 32 & 105 & 8 & 105 \\
\hline Pisani P-2006 & Asia(Thailand) & $211 / 408$ & 87 & 55 & 26 & 155 & 78 & 53 & 79 & 10 & 78 & 129 & 23 & 135 \\
\hline Yang MH-2007 & Asia(Korea) & $314 / 349$ & NA & NA & NA & NA & NA & NA & 116 & 16 & 182 & 111 & 18 & 220 \\
\hline Tao WH-2007 & Asia(china) & $47 / 94$ & 19 & 4 & 24 & 37 & 14 & 43 & NA & NA & NA & NA & NA & NA \\
\hline Cote ML-2007 & Mixed populations & $354 / 440$ & 80 & 5 & 269 & 95 & 6 & 339 & 19 & 0 & 326 & 34 & 6 & 400 \\
\hline Xia Y-2008 & Asia(china) & $58 / 116$ & 36 & 5 & 17 & 58 & 18 & 40 & NA & NA & NA & NA & NA & NA \\
\hline Qi XS-2008 & Asia(china) & $53 / 72$ & 29 & 7 & 17 & 38 & 11 & 23 & NA & NA & NA & NA & NA & NA \\
\hline Yoon KA-2008 & Asia(Korea) & $213 / 213$ & NA & NA & NA & NA & NA & NA & 76 & 10 & 127 & 87 & 10 & 116 \\
\hline Gallegos-Arreola-2008 & Mixed populations & $222 / 248$ & NA & NA & NA & NA & NA & NA & 91 & 40 & 91 & 104 & 11 & 133 \\
\hline Shah PP-2008 & Asia(India) & $200 / 200$ & & $94^{*}$ & 106 & & $63^{*}$ & 137 & & $67^{\#}$ & 133 & & $44^{\#}$ & 156 \\
\hline Kumar M-2009 & Asia(India) & $93 / 253$ & NA & NA & NA & NA & NA & NA & 17 & 3 & 73 & 40 & 3 & 210 \\
\hline Cote ML-2009 & Mixed populations & $502 / 523$ & 109 & 14 & 373 & 110 & 7 & 402 & 38 & 0 & 464 & 32 & 2 & 489 \\
\hline Honma HN-2009 & Mixed populations & $200 / 264$ & 76 & 11 & 113 & 94 & 9 & 161 & NA & NA & NA & NA & NA & NA \\
\hline Klinchid J-2009 & Asia(Thailand) & $85 / 82$ & & $66^{*}$ & 19 & & $66^{*}$ & 16 & & $47^{\#}$ & 33 & & $42^{\#}$ & 38 \\
\hline Timofeeva MN-2009 & Caucasians (German) & $619 / 1264$ & NA & NA & NA & NA & NA & NA & 248 & 61 & 260 & 545 & 117 & 585 \\
\hline Shaffi SM-2009 & Asia(India) & 109/163 & & $81^{*}$ & 28 & & $85^{*}$ & 78 & NA & NA & NA & NA & NA & NA \\
\hline Jin Y-2010 & Asia(China) & $124 / 154$ & & $71^{*}$ & 79 & & $70^{*}$ & 80 & NA & NA & NA & NA & NA & NA \\
\hline Wright CM-2010 & Caucasians (Australian) & 1040/784 & 219 & 24 & 797 & 128 & 10 & 646 & 103 & 8 & 929 & 40 & 3 & 741 \\
\hline
\end{tabular}

$\mathrm{NA}$, not applicable; *, the number of the combined of TypeB and TypeC genetypes; ${ }^{;}$, the number of the combined lle/Nal and Val/Val genotypes. 
Table 2 Summary ORs for various contrasts of CYP1A1 Mspl and exon7 gene polymorphisms in this meta-analysis

\begin{tabular}{|c|c|c|c|c|c|c|}
\hline \multirow[t]{2}{*}{ Subgroup analysis } & \multicolumn{3}{|c|}{ Mspl genotype } & \multicolumn{3}{|c|}{ exon7 genotype } \\
\hline & Contrast & studies & OR(95\%) $P_{h}$ & Contrast & studies & OR(95\%) $P_{h}$ \\
\hline Total & $\begin{array}{l}\text { Type C vs Type A } \\
\text { (TypeB+TypeC) vs Type A }\end{array}$ & 49 & $\begin{array}{l}1.26(1.12-1.42) 0.003 \\
1.20(1.13-1.28) 0.000\end{array}$ & $\begin{array}{l}\text { Val/Nal vs lle/lle } \\
\text { (lle/Nal +Val/Nal) vs Ile/lle }\end{array}$ & 40 & 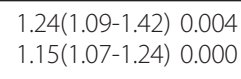 \\
\hline \multicolumn{7}{|l|}{ Ethnicity } \\
\hline Asian & $\begin{array}{l}\text { Type C vs Type A } \\
\text { (TypeB+TypeC) vs Type A }\end{array}$ & 26 & $\begin{array}{l}1.24(1.12-1.43) 0.004 \\
1.30(1.17-1.44) 0.002\end{array}$ & $\begin{array}{l}\text { Val/Nal vs lle/lle } \\
\text { (Ile/Nal +Val/Nal)vs Ile/lle }\end{array}$ & 22 & 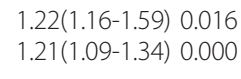 \\
\hline Caucasian & $\begin{array}{l}\text { Type C vs Type A } \\
\text { (TypeB+TypeC) vs Type A }\end{array}$ & 11 & $\begin{array}{l}1.25(1.09-1.36) 0.053 \\
1.35(1.18-1.54) 0.046\end{array}$ & $\begin{array}{l}\text { Val/Nal vs lle/lle } \\
\text { (Ile/Nal +Val/Nal) vs Ile/lle }\end{array}$ & 10 & 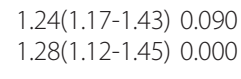 \\
\hline Mixed population & $\begin{array}{l}\text { Type C vs Type A } \\
\text { (TypeB+TypeC) vs Type A }\end{array}$ & 12 & $\begin{array}{l}1.05(0.89-1.28) 0.140 \\
1.02(0.92-1.14) 0.330\end{array}$ & $\begin{array}{l}\text { Val/Nal vs lle/lle } \\
\text { (lle/Nal +Val/Nal) vs Ile/lle }\end{array}$ & 8 & 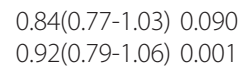 \\
\hline \multicolumn{7}{|l|}{ Histological type } \\
\hline SCC & $\begin{array}{l}\text { Type C vs Type A } \\
\text { (TypeB+TypeC) vs Type A }\end{array}$ & 13 & $\begin{array}{l}1.87(1.58-2.14) 0.005 \\
1.93(1.62-2.30) 0.000\end{array}$ & $\begin{array}{l}\text { Val/Nal vs lle/lle } \\
\text { (lle/Nal +Val/Nal) vs Ile/lle }\end{array}$ & 11 & $\begin{array}{l}1.38(1.12-1.66) 0.004 \\
1.42(1.18-1.70) 0.007\end{array}$ \\
\hline$A C$ & $\begin{array}{l}\text { Type C vs Type A } \\
\text { (TypeB+TypeC) vs Type A }\end{array}$ & 12 & $\begin{array}{l}1.34(1.14-1.56) 0.014 \\
1.20(1.01-1.43) 0.000\end{array}$ & $\begin{array}{l}\text { Val/Nal vs lle/lle } \\
\text { (lle/Nal +Val/Nal) vs lle/lle }\end{array}$ & 10 & 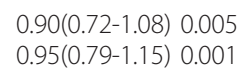 \\
\hline $\mathrm{SCLC}$ & $\begin{array}{l}\text { Type C vs Type A } \\
\text { (TypeB+TypeC) vs Type A }\end{array}$ & 8 & $\begin{array}{l}0.96(0.70-1.26) 0.864 \\
1.06(0.77-1.45) 0.976\end{array}$ & $\begin{array}{l}\text { Val/Nal vs lle/lle } \\
\text { (Ile/Nal + Val/Nal) vs lle/lle }\end{array}$ & 7 & $\begin{array}{l}0.84(0.68-1.08) 0.068 \\
0.78(0.53-1.14) 0.039\end{array}$ \\
\hline \multicolumn{7}{|l|}{ Gender } \\
\hline Male & $\begin{array}{l}\text { Type C vs Type A } \\
\text { (TypeB+TypeC) vs Type A }\end{array}$ & 3 & $\begin{array}{l}1.39(1.23-1.79) 0.210 \\
1.46(1.07-1.98) 0.380\end{array}$ & $\begin{array}{l}\text { Val/Nal vs lle/lle } \\
\text { (lle/Nal +Val/Nal) vs Ile/lle }\end{array}$ & 7 & 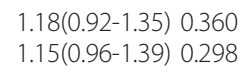 \\
\hline Female & $\begin{array}{l}\text { Type C vs Type A } \\
\text { (TypeB+TypeC) vs Type A }\end{array}$ & 7 & $\begin{array}{l}0.92(0.84-1.16) 0.003 \\
0.85(0.71-1.02) 0.000\end{array}$ & $\begin{array}{l}\text { Val/Nal vs lle/lle } \\
\text { (lle/Nal +Val/Nal) vs Ile/lle }\end{array}$ & 3 & $\begin{array}{l}1.29(1.08-1.51) 0.000 \\
1.24(1.05-1.47) 0.002\end{array}$ \\
\hline Smoking status & & 13 & & & 10 & \\
\hline Smokers & $\begin{array}{l}\text { Type C vs Type A } \\
\text { (TypeB+TypeC) vs Type A }\end{array}$ & & $\begin{array}{l}1.62(1.33-1.96) 0.000 \\
1.75(1.44-2.13) 0.003\end{array}$ & $\begin{array}{l}\text { ValNal vs lle/lle } \\
\text { (Ile/Nal + Val/Nal) vs lle/lle }\end{array}$ & & 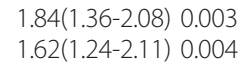 \\
\hline Non-smokers & $\begin{array}{l}\text { Type C vs Type A } \\
\text { (TypeB+TypeC) vs Type A }\end{array}$ & & $\begin{array}{l}1.18(0.96-1.48) 0.086 \\
1.09(0.90-1.33) 0.114\end{array}$ & $\begin{array}{l}\text { Val/Nal vs lle/lle } \\
(\text { Ile/Nal +Val/Nal) vs lle/lle }\end{array}$ & & $\begin{array}{l}1.18(0.96-1.38) 0.080 \\
1.07(0.88-1.31) \\
0.002\end{array}$ \\
\hline
\end{tabular}

$\mathrm{P}_{\mathrm{h}} \mathrm{P}$ value of $\mathrm{Q}$-test for heterogeneity test

was also observed in Caucasian carriers of Val/Val vs Ile/ Ile $(\mathrm{OR}=1.24 ; 95 \% \mathrm{CI}=1.17-1.43 ; P=0.090$ for heterogeneity) and Ile/Val and Val/Val combined vs Ile/Ile (OR = $1.28 ; 95 \% \mathrm{CI}=1.12-1.45 ; P=0.000$ for heterogeneity). However, no significant associations were observed in mixed populations for both $\mathrm{Val} / \mathrm{Val}$ vs Ile/Ile $(\mathrm{OR}=0.84$; $95 \% \mathrm{CI}=0.77-1.03 ; P=0.090$ for heterogeneity) or Ile/Val and $\mathrm{Val} / \mathrm{Val}$ combined vs Ile/Ile $(\mathrm{OR}=0.92 ; 95 \% \mathrm{CI}=$ $0.79-1.06 ; P=0.001$ for heterogeneity) (Table 2).

Twelve [22,24,29-32,36,40,53,57,58,70] out of 64 studies examined the association of CYP1A1 exon 7 genotype and the risk of different histological types of lung cancer including SCC, AC and SCLC. Among lung SCC, significantly increased risks were observed for both $\mathrm{Val} / \mathrm{Val}$ vs Ile/Ile $(\mathrm{OR}=1.38 ; 95 \% \mathrm{CI}=1.12-1.66 ; P=0.004$ for heterogeneity) or Ile/Val and Val/Val combined vs Ile/Ile $(\mathrm{OR}=1.42 ; 95 \% \mathrm{CI}=1.18-1.70 ; P=0.007$ for heterogeneity. However, among lung AC and SCLC, no significant associations were observed for both $\mathrm{Val} / \mathrm{Val}$ vs Ile/lle or Ile/Val and Val/Val combined vs Ile/Ile (Figure 7).

Eight $[36,54,56,57,70,74,76,77]$ out of 64 studies included the association of CYP1A1 exon 7 genotype and lung caner risk stratified by gender (Male and Female). For Female population (3 studies), significantly increased risks were observed for both $\mathrm{Val} / \mathrm{Val}$ vs Ile/Ile $(\mathrm{OR}=$
$1.29 ; 95 \% \mathrm{CI}=1.08-1.51 ; P=0.000$ for heterogeneity), Ile/Val and $\mathrm{Val} / \mathrm{Val}$ combined vs Ile/Ile $(\mathrm{OR}=1.24 ; 95 \%$ $\mathrm{CI}=1.05-1.47 ; P=0.002$ for heterogeneity). However, for Male population (7 studies), no significant associations were observed for both $\mathrm{Val} / \mathrm{Val}$ vs Ile/Ile $(\mathrm{OR}=$ 1.18 ; $95 \% \mathrm{CI}=0.92-1.35 ; P=0.360$ for heterogeneity) or Ile/Val and $\mathrm{Val} / \mathrm{Val}$ combined vs Ile/Ile (OR $=1.15 ; 95 \%$ $\mathrm{CI}=0.96-1.39 ; P=0.298$ for heterogeneity) (Figure 8).

Ten $[24,31,56,60,70-73]$ out of 64 studies included the association of CYP1A1 exon 7 genotype and lung caner risk stratified by smoking status (non-smokers or never smokers and smokers). For smokers, significantly increased risks were observed for both $\mathrm{Val} / \mathrm{Val}$ vs Ile/lle $(\mathrm{OR}=1.84 ; 95 \% \mathrm{CI}=1.36-2.08 ; P=0.003$ for heterogeneity), Ile/Val and $\mathrm{Val} / \mathrm{Val}$ combined vs Ile/Ile (OR = 1.62; $95 \% \mathrm{CI}=1.24-2.11 ; P=0.004$ for heterogeneity). However, for non-smokers, no significant associations were observed for both $\mathrm{Val} / \mathrm{Val}$ vs Ile/Ile $(\mathrm{OR}=1.18$; 95\% CI $=0.96-1.38 ; P=0.080$ for heterogeneity) or Ile/ $\mathrm{Val}$ and $\mathrm{Val} / \mathrm{Val}$ combined vs Ile/Ile $(\mathrm{OR}=1.07 ; 95 \%$ $\mathrm{CI}=0.88-1.31 ; P=0.002$ for heterogeneity) (Figure 9).

\subsection{Sensitivity analyses}

On omission of each individual study, the corresponding pooled OR was not altered materially (data not shown). 


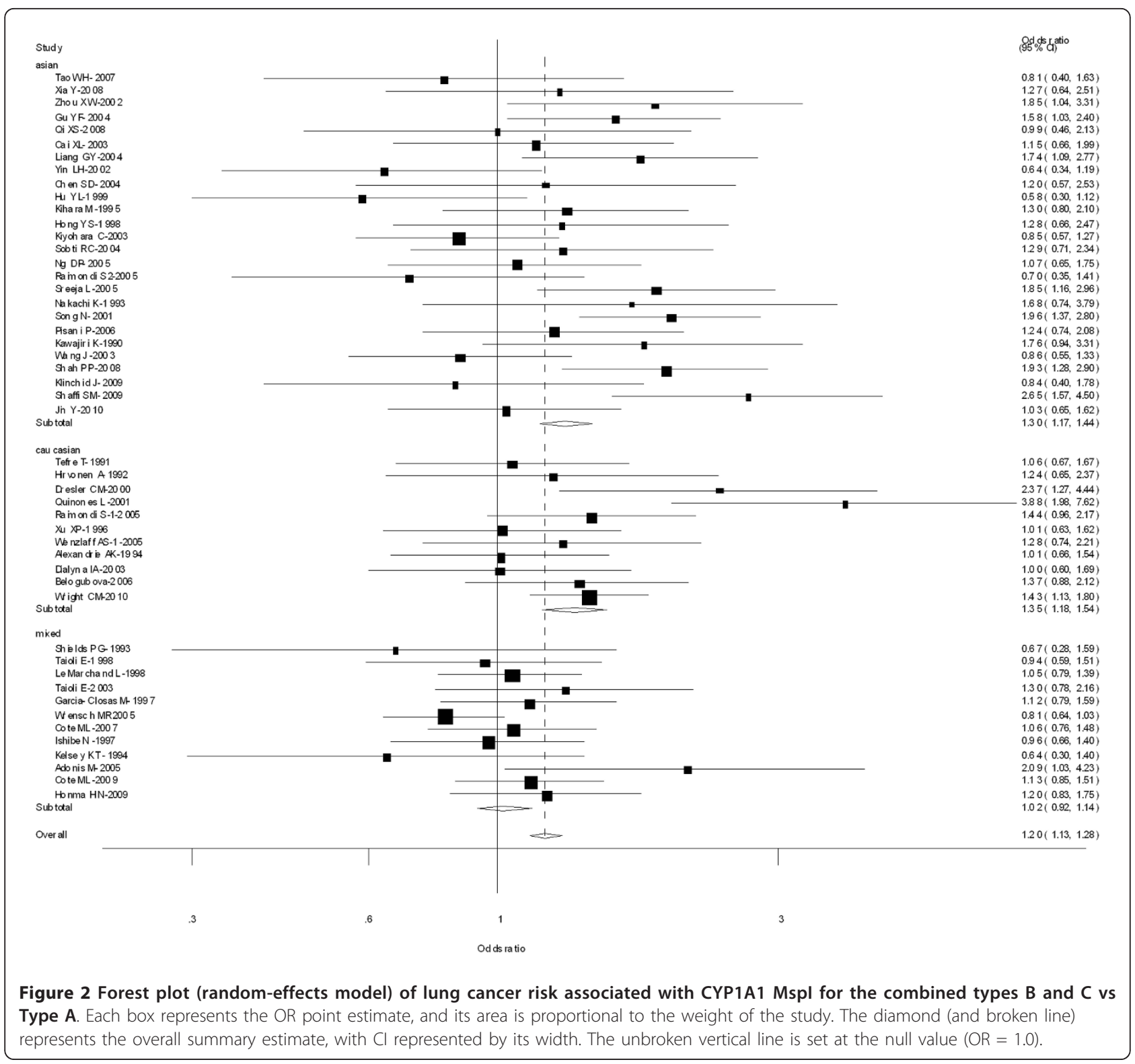

\subsection{Publication bias}

Begg's funnel plot and Egger's test were performed to identify any publication bias. The funnel plots did not exhibit any patent asymmetry (Figure 10 and 11 ). By Egger's test-used to provide statistical evidence of funnel plot symmetry-there was no evidence of publication bias $(\mathrm{P}=0.558$ for publication bias of MspI and $\mathrm{P}=$ 0.722 for publication bias of exon 7).

\section{Discussion}

CYP genes are large families of endoplasmic and cytosolic enzymes that catalyze the activation and detoxification, respectively, of reactive electrophilic compounds, including many environmental carcinogens (e.g., benzo[a] pyrene). CYP1A1 is a phase I enzyme that regulates the metabolic activation of major classes of tobacco procarcinogens, such as aromatic amines and PAHs [6]. Thus, it might affect the metabolism of environmental carcinogens and alter the susceptibility to lung cancer. This meta-analysis explored the association between the CYP1A1 MspI and exon7 gene polymorphisms and lung cancer risk, and performed the subgroup analysis stratified by ethnicity, histological types of lung caner, gender and smoking status of case and control population. Our results indicated a significant association between CYP1A1 MspI gene polymorphism and lung cancer risk in Asians, Caucasians, lung SCC, lung $\mathrm{AC}$ and Male population, no significant association was found in mixed population, lung SCLC 


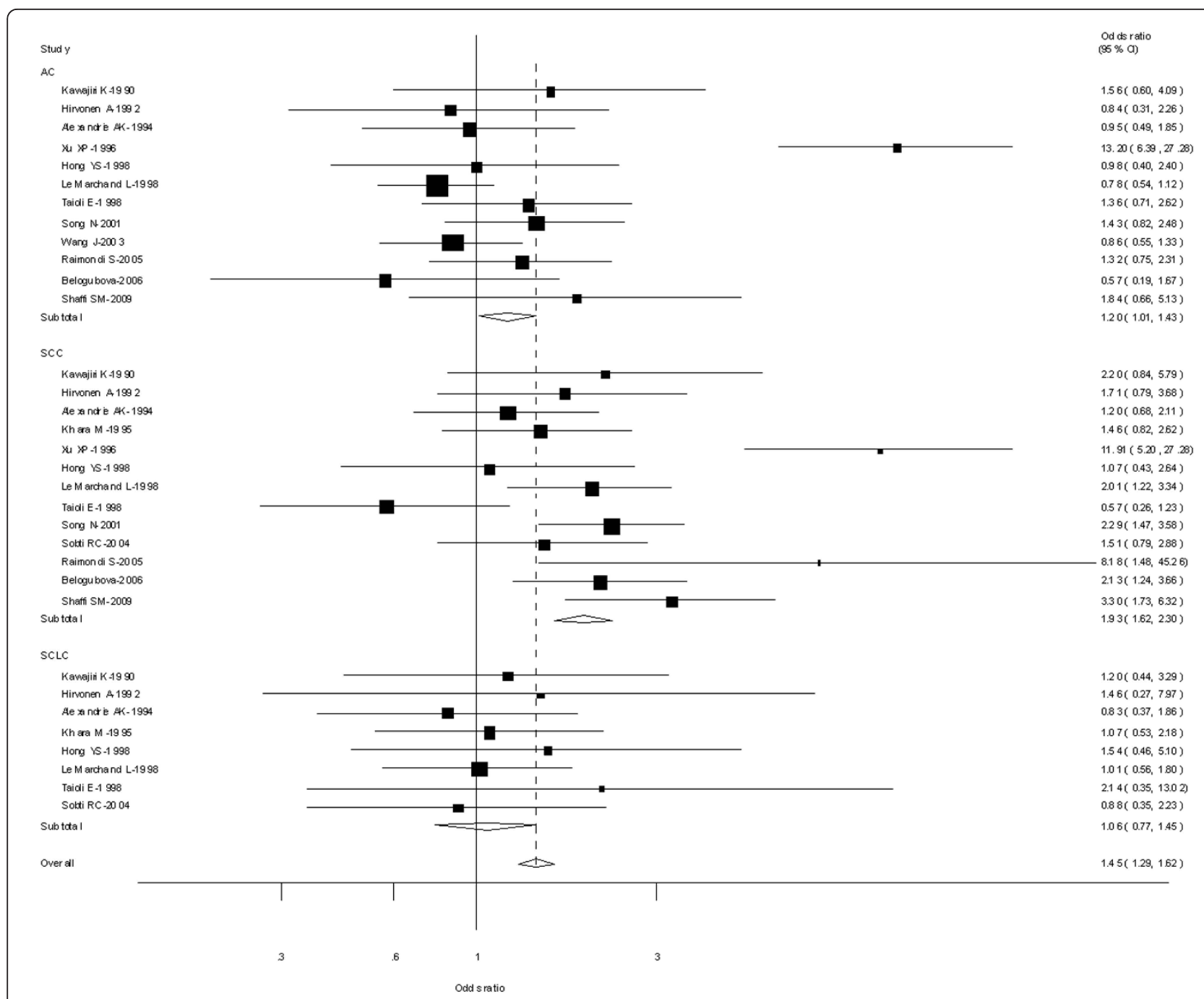

Figure 3 Forest plot (random-effects model) of lung cancer risk associated with CYP1A1 Mspl for the combined types B and C vs Type A stratified by histological types of lung cancer.

and Female population. Interestingly, inconsistent results were observed for CYP1A1 exon7 polymorphism in our meta-analysis. For the association between CYP1A1 exon7 gene polymorphism and lung cancer risk, a significant assocation was found in Asians, Caucasians, lung SCC and Female population, no significant associations were found in mixed population, lung AD, lung SCLC and Male population. Additionally, a significant association was found in smoker population and not in non-smoker populations for CYP1A1 MspI and exon7 polymorphisms.

When stratified according to ethnicity, a significantly increased risks were identified among Asians and Caucasians for the $2 \mathrm{MspI}$ genotype variants, however no significant association was found in mixed population. For exon 7 polymorphism, the same risk was found in Asians and Caucasians, not in mixed population. These findings indicate that polymorphisms of CYP1A1 MspI and exon 7 polymorphism may be important in specific ethnicity of lung cancer patients. Population stratification is an area of concern, and can lead to spurious evidence for the association between the marker and disease, suggesting a possible role of ethnic differences in genetic backgrounds and the environment they lived in [81]. In fact, the distribution of the less common Val allele of exon 7 genotype varies extensively between different races, with a prevalence of $\sim 25 \%$ among East Asians, 5\% among Caucasians and $\sim 15 \%$ among other population. In addition, in our meta-analysis the between-study heterogeneity was existed in overall population, the subgroup of Asian and Caucasian for MspI and exon 7 genotypes. Therefore, additional studies are warranted to further validate ethnic difference in the effect of this functional polymorphism on lung cancer risk. 


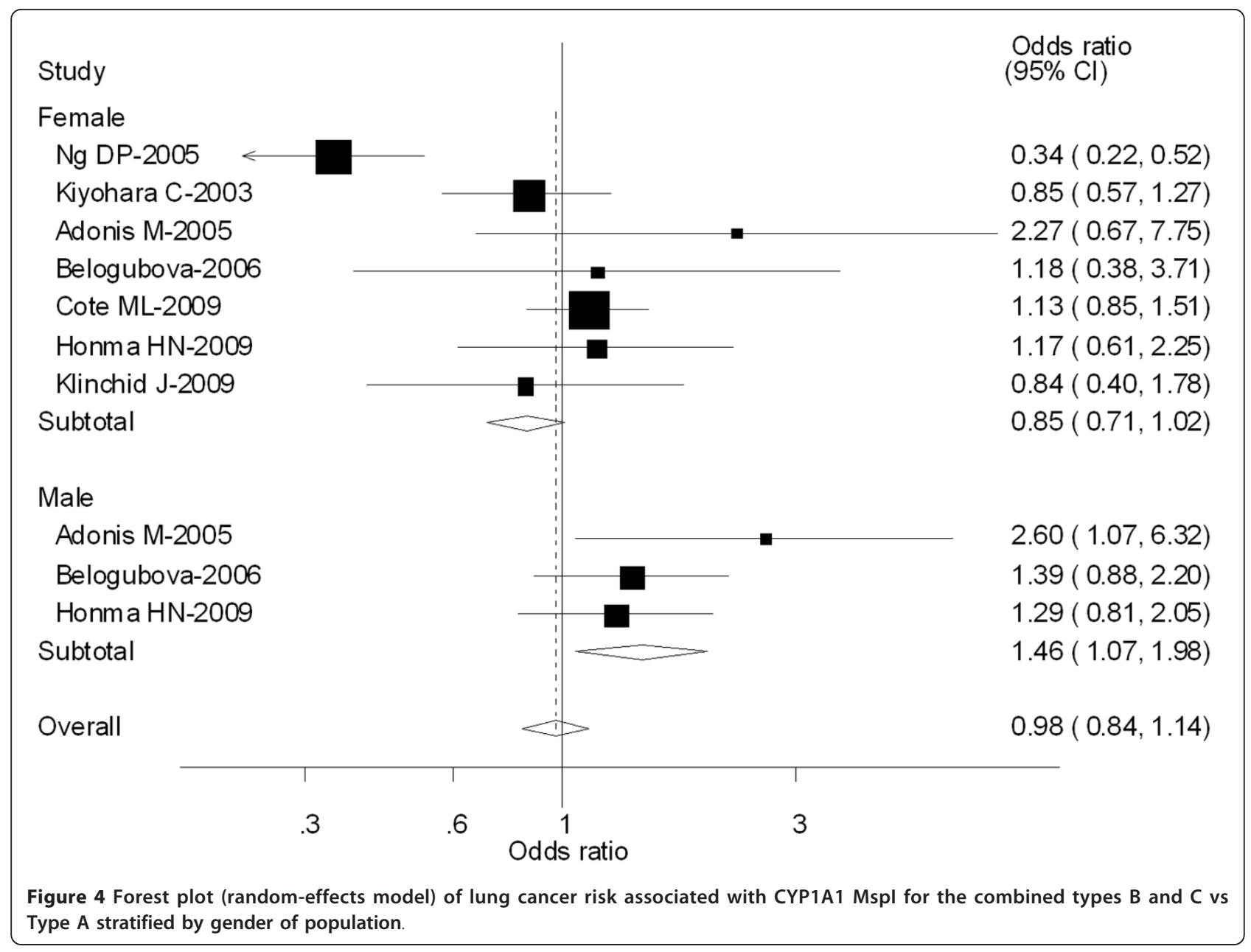

There are growing biological and epidemiological data to suggest that different lung cancer pathological subtypes, particularly the two most common, are distinct etiological entities that should be analyzed separately [82]. When subgroup analyses by pathological types were considered, CYPIAl Mspl and exon7 variant alleles were found to be associated with a 1.4-1.9 fold increase in the risk of lung SCC. For lung AC, only CYPIAl Mspl gene polymorphism was significant, however, for lung SCLC, no significant association was found for two genotypes. Our findings were consistent with the Le Marchand L et al study [32] with largest sample sizes of case and control. Le Marchand et al. [32] hypothesized that genetic susceptibility to PAHs predominantly caused lung SCC and nitrosamines caused lung AC. With introduction of filter-tipped cigarettes, probably decreased smokers' exposure to PAHs and increased their exposure to nitrosamines, decreasing trend of SCC, relative to the increase in AC indirectly supports this hypothesis [83]. Different carcinogenic processes may be involved in the genesis of various tumor types because of the presence of functionally different CYP1Al Mspl and exon7 gene polymorphisms. However, the possible molecular mechanisms to explain these histology-specific differences in the risk of lung cancer remain unresolved.

Recent epidemiological and biochemical studies have suggested increased susceptibility to tobacco carcinogens in women compared to men [84-86]. Moreover, CYP1A1 mRNA expression in the lung has been observed to be more than two-fold higher in female smokers compared with male smokers [87]. Another possibly was due to the effect of circulation estrogens, which have been shown to induce expression of PAH-metabolizing enzymes, such as CYP1A1, thereby increasing metabolic activation of carcinogens [88]. In premenopausal women, a higher expression of estrogen can be expected. Estrogen by itself can be involved in carcinogenesis and additionally, it can stimulate expression of CYPs in the female. In our metaanalysis, we found that the effect of CYP1A1 exon7 genotype was observed only in Females, however, for CYP1A1 Mspl the effect was only observed among Males. Our results, along with the previous studies involved above, suggest the difference roles on the two polymorphisms of 


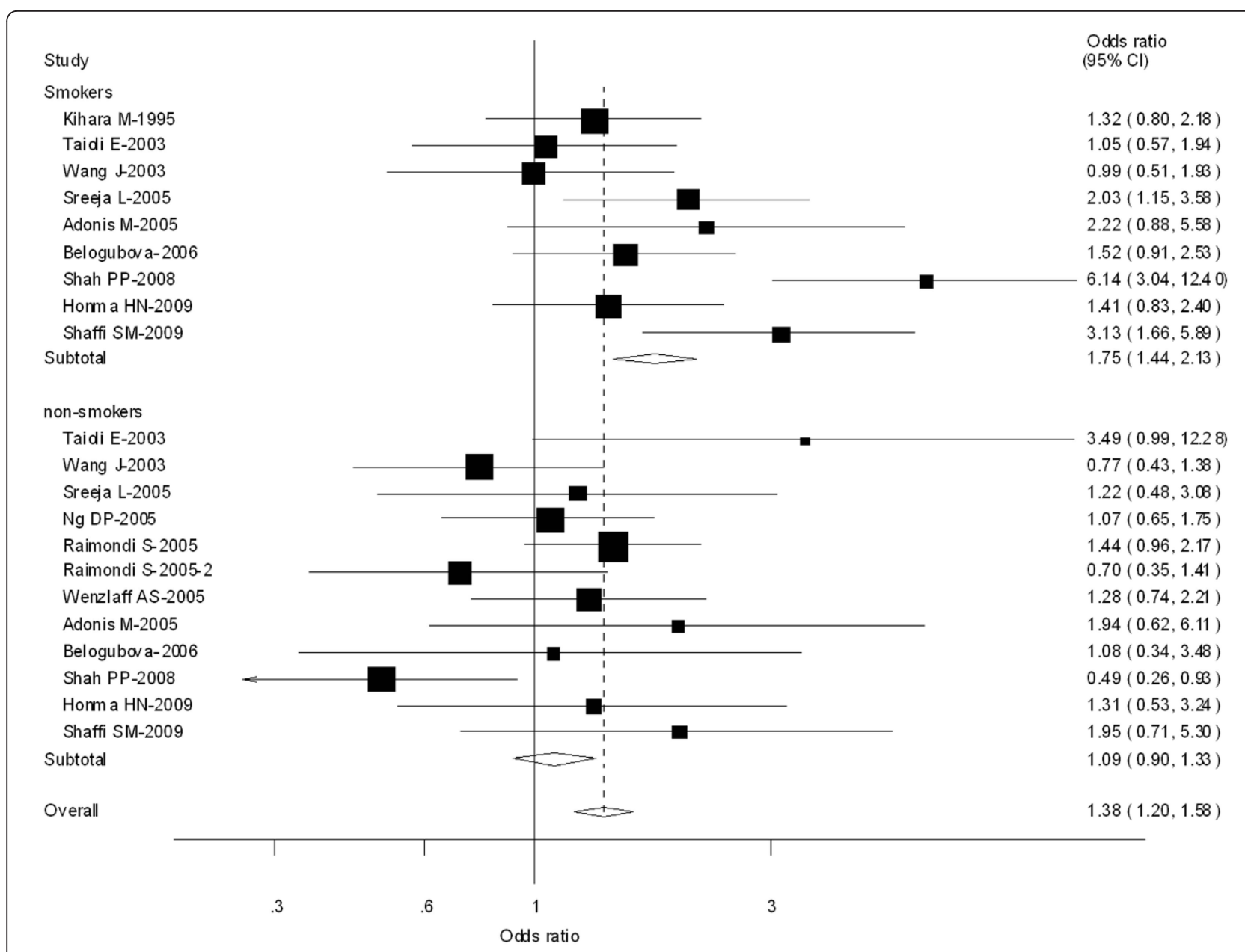

Figure 5 Forest plot (random-effects model) of lung cancer risk associated with CYP1A1 Mspl for the combined types B and C vs Type A stratified by smoking status of population.

CYP1A1 genotypes in the susceptibility of lung cancer between Females and Males.

As we know, aside from genetic factor, smoking is the major risk factor of lung cancer. Most studies out of 64 studies reported information on smoking habits of cases and controls, however only sixteen eligible publications provided non-smokers information. Our meta-analysis results showed that a significantly increased risk was found to be associated with the CYP1A1 MspI and exon 7 gene polymorphisms and lung cancer risk in smokers, however, no significant association was found among nonsmokers neither CYP1A1 MspI or exon 7 genotype. Tobacco smoke contains many of carcinogens and procarcinogens, such as benzopyrene and nitrosamine. These compounds are metabolized by the phase I enzymes including CYP family enzymes and converted to inactivemetabolites by the phase II enzymes. Our results should indicate the interaction between CYP1A1 MspI and exon 7 gene polymorphisms and smoking in the development of lung carcinoma. However, the association between the extent of smoke exposure and lung caner risk was not clear, further studies with larger sample size are needed to provide insights into the association.

Our data were consistent with the primary results of a previous meta-analysis [89] that showed the MspI and Ile-Val polymorphism of CYP1A1 was a risk factor associated with increased lung cancer susceptibility and these associations varied in different ethnic populations. However, that meta-analysis only conducted the stratified analysis according to ethnicity, smoking and histological types and could not analyze the stratified results in-depth. They could not certify the interaction between smoking status, the major risk fact of lung cancer, and the two genotypes of CYP1A1 polymorphism due to the limitation of included studies. We performed more comprehensive stratified analysis by ethnicity, histological types, smoking status and gender and found the different associations in Male and Female population. We concluded 


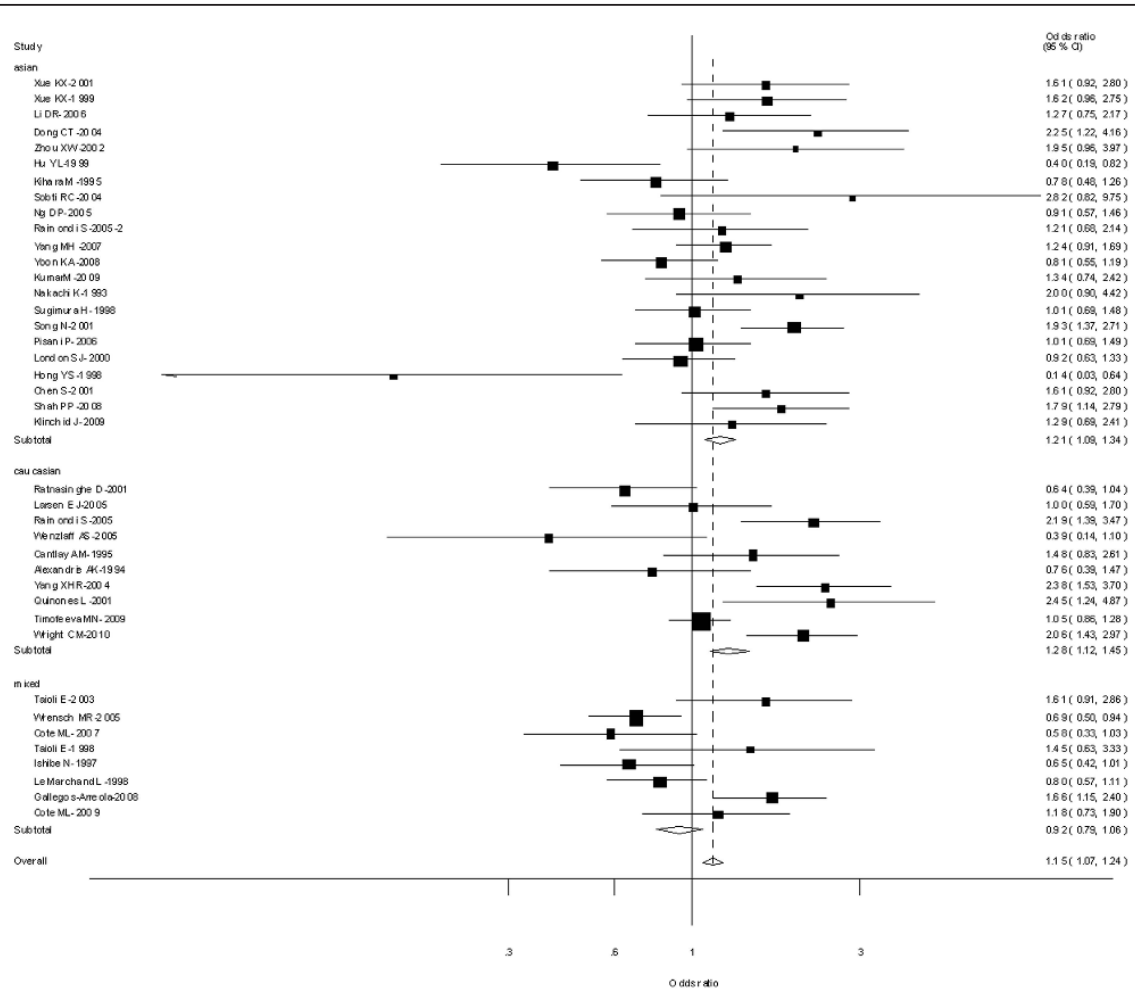

Figure 6 Forest plot (random-effects model) of lung cancer risk associated with CYP1A1 exon7 genotype for the combined lle/Val and Val/Val vs Ile/lle.

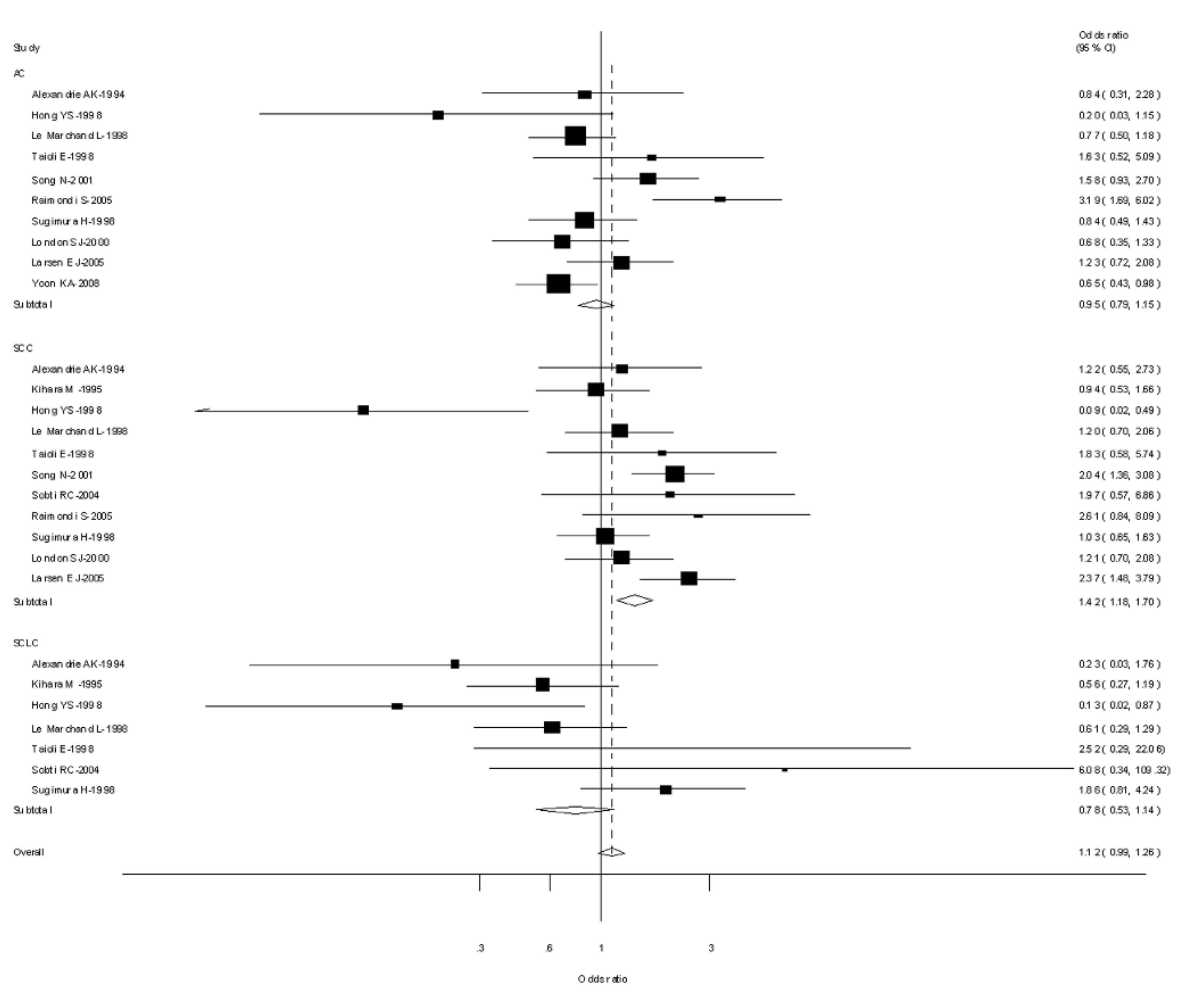

Figure 7 Forest plot (random-effects model) of lung cancer risk associated with CYP1A1 exon7 genotype for the combined Ile/Val and $\mathrm{Val} / \mathrm{Val}$ vs Ile/lle by histological types of lung cancer. 


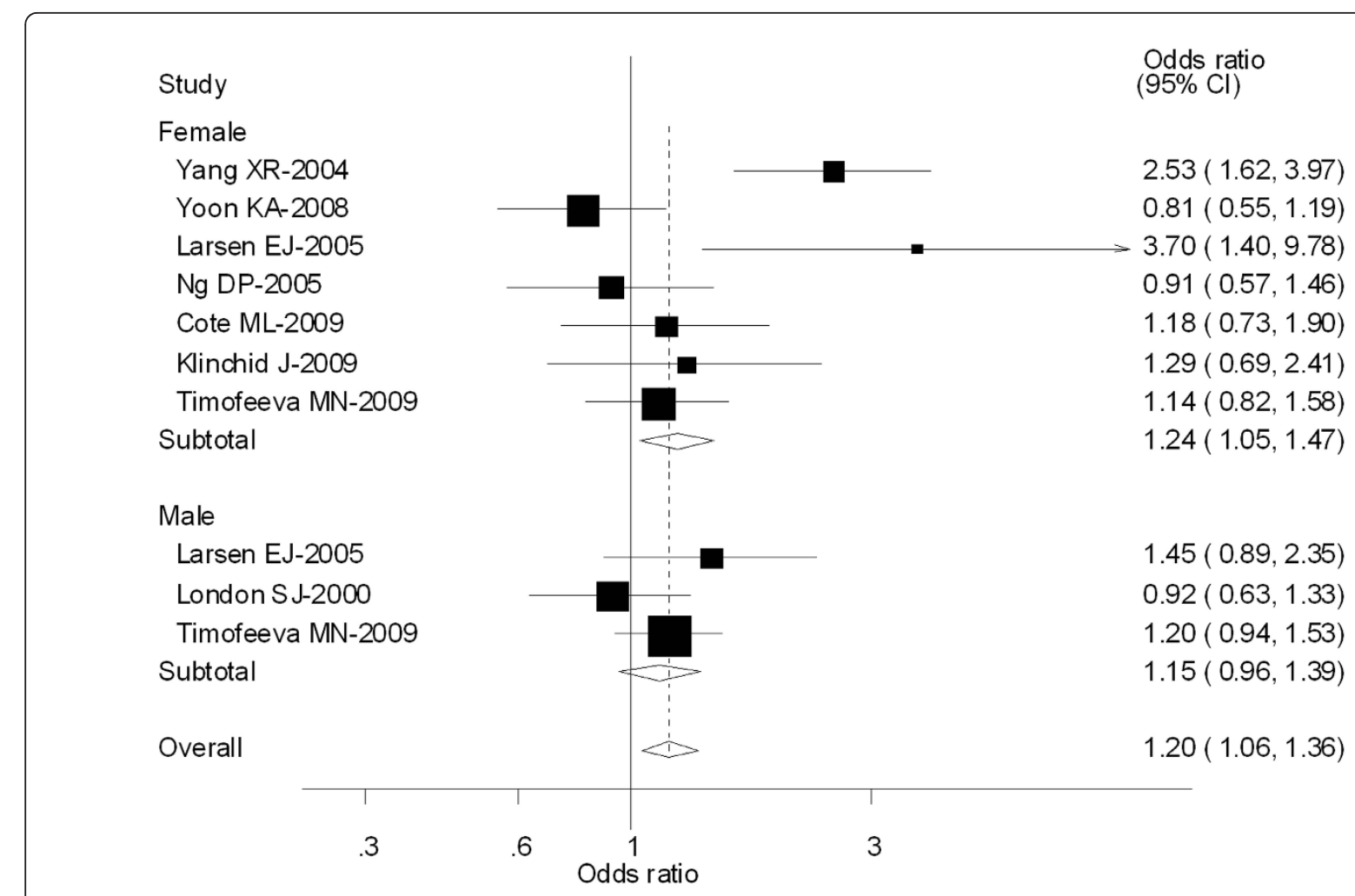

Figure 8 Forest plot (random-effects model) of lung cancer risk associated with CYP1A1 exon7 genotype for the combined lle/Val and $\mathrm{Val} / \mathrm{Val}$ vs lle/lle stratified by gender of population.

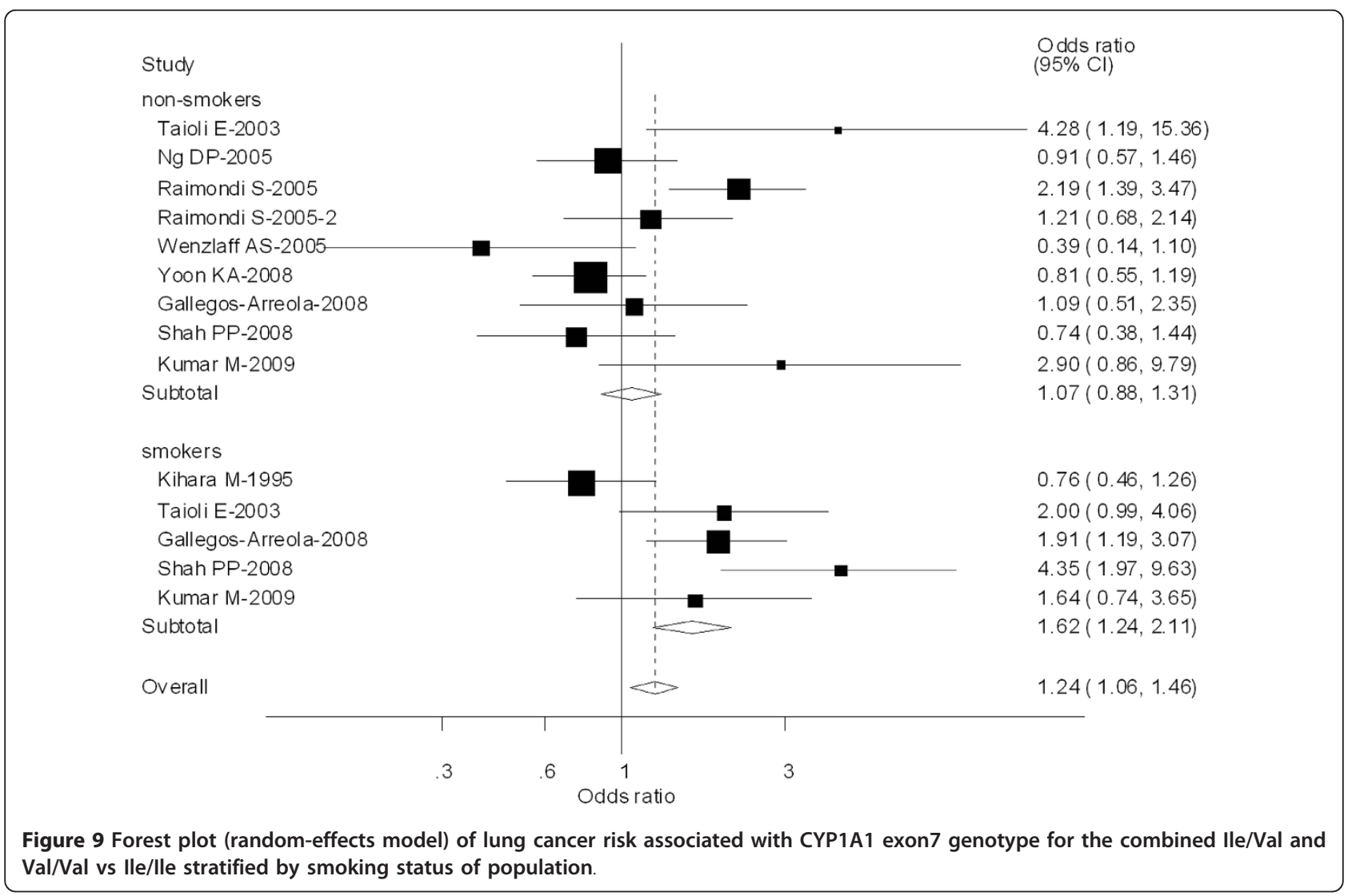




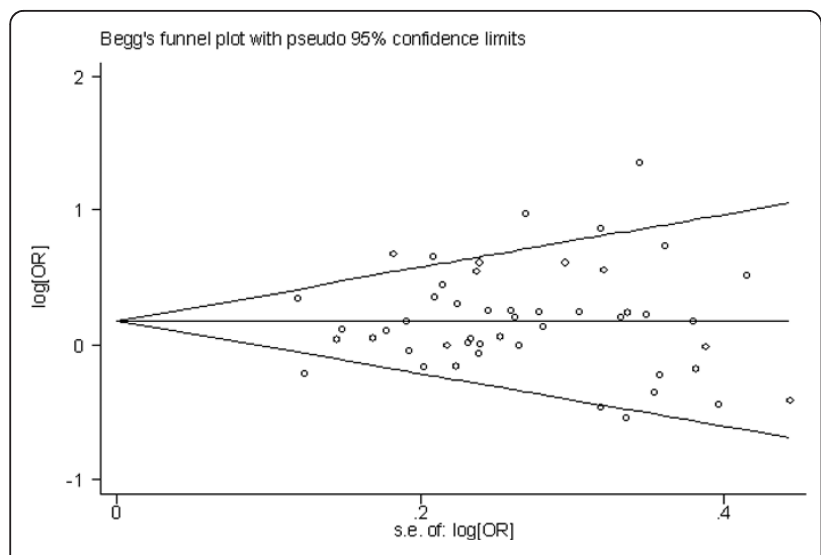

Figure 10 Begg's funnel plot of CYP1A1 Mspl gene polymorphism and lung cancer risk for the combined types B and $C$ vs Type $A$.

that MspI and exon 7 polymorphisms of CYP1A1 correlated with increased lung cancer susceptibility and there was an interaction between two genotypes of CYP1A1 polymorphism and smoking, but these associations varied in different ethnic populations, histological types and gender of case and control population.

Some limitations of this meta-analysis should be acknowledged. First, heterogeneity can interfere with the interpretation of the results of a meta-analysis. Although we minimized this likelihood by performing a careful search of published studies, using explicit criteria for a study's inclusion and performing strict data extraction and analysis, significant interstudy heterogeneity nevertheless existed in nearly every comparison. The presence of heterogeneity can result from differences in the selection of controls, age distribution, and prevalence of lifestyle factors. Further, only published studies were included in this meta-analysis. The presence of publication bias indicates that non-significant or negative

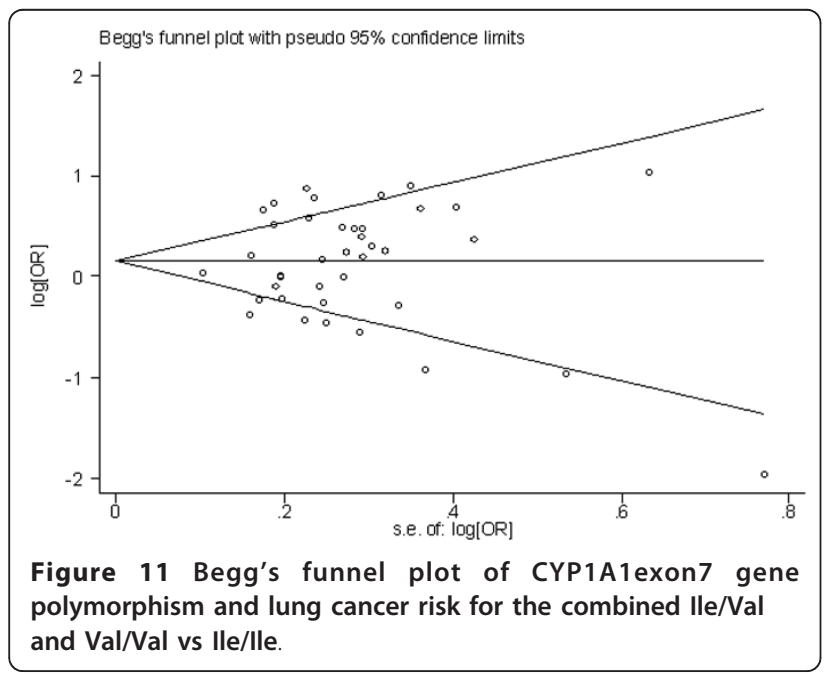

findings might be unpublished. Finally, in the subgroup analyses, different ethnicities were confused with other population, which may bring in some heterogeneity. As studies among the Indians and Africans are currently limited, further studies including a wider spectrum of subjects should be carried to investigate the role of these variants in different populations.

In conclusion, the results of our meta-analysis have provided the comprehensive and convincing evidence that CYP1A1 MspI and exon 7 polymorphisms are an important modifying factor in determining susceptibility to lung cancer. The effect of two genotypes of CYP1A1 polymorphism is diverse by the subgroup analysis stratified by ethnicity, histological types of lung caner and gender of case and control population. More importantly, our study confirms that there is an interaction between two genotypes of CYP1A1 polymorphism and smoking. For future studies, strict selection of patients, wellmatched controls and larger sample size will be required. Moreover, gene-gene and gene-environment interactions should also be considered.

\section{List of abbreviations}

CYP1A1: Cytochrome P450 1A1; PAHs: polycyclic aromatic hydrocarbons; CNKI: China National Knowledge Infrastructure; SCC: squamous carcinoma; AC: adenocarcinoma; SCLC: small cell lung cancer; OR: odds ratios; Cl: confidence interval

\section{Acknowledgements}

This work was supported in part by a grant from the Major Program of Nanjing Medical Science and Technique Development Foundation (Molecular Predictor of Personalized Therapy for Chinese Patients with Nonsmall Cell Lung Cancer) (Lk-Yu).

\section{Author details}

${ }^{1}$ First Department of Respiratory Medicine, Nanjing Chest Hospital, 215 Guangzhou Road, Nanjing 210029, China. ${ }^{2}$ Department of Respiratory Medicine, No. 81 Hospital of PLA, Nanjing, China. ${ }^{3}$ Department of Respiratory Medicine, Jinling Hospital, Nanjing University School of Medicine, Nanjing, China.

\section{Authors' contributions}

PZ and LKY contributed to the conception and design of the study, the analysis and interpretation of data, the revision of the article as well as final approval of the version to be submitted. SZW and QQ participated in the design of the study, performed the statistical analysis, searched and selected the trials, drafted and revised the article. QW participated in the design of the study and helped to revise the article. All authors read and approved the final version of the manuscript.

\section{Competing interests}

The authors declare no any conflicts of interest in this work.

Received: 8 September 2011 Accepted: 20 October 2011

Published: 20 October 2011

\section{References}

1. Alberg AJ, Samet JM: Epidemiology of lung cancer. Chest 2003, 123:21-49.

2. Molina JR, Yang P, Cassivi SD, Schild SE, Adjei AA: Non-small cell lung cancer: epidemiology, risk factors, treatment, and survivorship. Mayo Clin Proc 2008, 83:584-594.

3. Alberg AJ, Brock MV, Samet JM: Epidemiology of lung cancer: looking to the future. J Clin Oncol 2005, 23:3175-85. 
4. Rodriguez V, Tardon A, Kogevinas M, Prieto CS, Cueto A, Garcia M Menendez IA, Zaplana J: Lung cancer risk in iron and steel foundry workers: a nested case control study in Asturias, Spain. Am J Ind Med 2000, 38:644-50.

5. Tardon A, Lee WJ, Delgado-Rodriguez M, Dosemeci M, Albanes D, Hoover R, Blair A: Leisure-time physical activity and lung cancer: a metaanalysis. Cancer Causes Control 2005, 16:389-97.

6. Guengerich FP, Shimada T: Activation of procarcinogens by human cytochrome P450 enzymes. Mutat Res 1998, 400:201-213.

7. Butler JP, Post GB, Lioy PJ, Waldman JM, Greenberg A: Assessment of carcinogenic risk from personal exposure to benzo[a]pyrene in the total human environmental exposure study(THEES). J Air Waste Manag Assoc 1993, 43:970-977.

8. Kawajiri K, Eguchi H, Nakachi K, Sekiya T, Yamamoto M: Association of CYP1A1 germ line polymorphisms with mutations of the p53 gene in lung cancer. Cancer Res 1996, 56:72-76.

9. Kawajiri K, Nakachi K, Imai K, Yoshii A, Shinoda N, Watanabe J: Identification of genetically high risk individuals to lung cancer by DNA polymorphisms of the cytochrome P450IA1 gene. FEBS 1990, 1:131-133.

10. Houlston RS: CYP1A1 polymorphisms and lung cancer risk: a metaanalysis. Pharmacogenetics 2000, 10(2):105-14.

11. Le Marchand L, Guo C, Benhamou S, Bouchardy C, Cascorbi I, Clapper ML, Garte S, Haugen A, Ingelman-Sundberg M, Kihara M, Rannug A, Ryberg D, Stücker I: Pooled analysis of the CYP1A1 exon 7 polymorphism and lung cancer (United States). Cancer Causes Control 2003, 14(4):339-46.

12. Shi X, Zhou S, Wang Z, Zhou Z, Wang Z: CYP1A1 and GSTM1 polymorphisms and lung cancer risk in Chinese populations: a metaanalysis. Lung Cancer 2008, 59(2):155-63.

13. Cochran WG: The combination of estimates from different experiments. Biometrics 1954, 10:101-29.

14. Mantel N, Haenszel W: Statistical aspects of the analysis of data from retrospective studies of disease. J Natl Cancer Inst 1959, 22:719-48.

15. DerSimonian R, Laird N: Meta-analysis in clinical trials. Control Clin Trials 1986, 7:177-88.

16. Tobias A: Assessing the influence of a single study in the meta-analysis estimate. Stata Tech Bull 1999, 8:15-7.

17. Egger M, Davey Smith G, Schneider M, Minder C: Bias in metaanalysis detected by a simple, graphical test. BMJ 1997, 315:629-34.

18. Tefre T, Ryberg D, Haugen A, Nebert DW, Skaug V, Brogger A, Borresen AL Human CYP1A1 (cytochrome P450) gene: lack of association between the Mspl restriction fragment length polymorphism and incidence of lung cancer in a Norwegian population. Pharmacogenetics 1991, 1:20-25.

19. Hirvonen A, Husgafvel-Pursiainen K, Karjalainen A, Anttila S, Vainio H: Pointmutational Mspl and Ile-Val polymorphisms closely linked in the CYP1A1 gene: lack of association with susceptibility to lung cancer in a Finnish study population. Cancer Epidemiol Biomarkers Prev 1992, 1:485-9.32.

20. Shields PG, Caporaso NE, Falk RT, Sugimura H, Trivers GE, Trump BF, Hoover RN, Weston A, Harris CC: Lung cancer, race, and a CYP1A1 genetic polymorphism. Cancer Epidemiol Biomarkers Prev 1993, 2(5):481-5.

21. Nakachi K, Imai K, Hayashi S, Kawajiri K: Polymorphisms of the CYP1A1 and glutathione S-transferase genes associated with susceptibility to lung cancer in relation to cigarette dose in a Japanese population. Cancer Res 1993, 53:2994-9.

22. Alexandrie AK, Sundberg MI, Seidegård J, Tornling G, Rannug A: Genetic susceptibility to lung cancer with special emphasis on CYP1A1 and GSTM1: a study on host factors in relation to age at onset, gender and histological cancer types. Carcinogenesis 1994, 15:1785-90.

23. Kelsey KT, Wiencke JK, Spitz MR: A race-specific genetic polymorphism in the CYP1A1 gene is not associated with lung cancer in African Americans. Carcinogenesis 1994, 15:1121-4

24. Kihara M, Kihara M, Noda K: Risk of smoking for squamous and small cell carcinomas of the lung modulated by combinations of CYP1A1 and GSTM1 gene polymorphisms in a Japanese population. Carcinogenesis 1995, 16:2331-6.

25. Cantlay AM, Lamb D, Gillooly M, Norrman J, Morrison D, Smith CA, Harrison DJ: Association between the CYP1A1 gene polymorphism and susceptibility to emphysema and lung cancer. Clin Mol Pathol 1995, 48: M210-M214

26. Xu X, Kelsey KT, Wiencke JK, Wain JC, Christiani DC: Cytochrome P450 CYP1A1 Mspl polymorphism and lung cancer susceptibility. Cancer Epidemiol Biomarkers Prev 1996, 5:687-92.
27. Ishibe N, Wiencke JK, Zuo ZF, McMillan A, Spitz M, Kelsey KT: Susceptibility to lung cancer in light smokers associated with CYP1A1 polymorphisms in Mexican and African-Americans. Cancer Epidemiol Biomarkers Prev 1997, 6:1075-80.

28. Garcia-Closas M, Kelsey KT, Wiencke JK, Xu X, Wain JC, Christiani DC: A case-control study of cytochrome P450 1A1, glutathione S-transferase M1, cigarette smoking and lung cancer susceptibility (Massachusetts, United States). Cancer Causes Control 1997, 8(4):544-53.

29. Hong YS, Chang JH, Kwon OJ, Ham YA, Choi JH: Polymorphism of the CYP1A1 and glutathione-S-transferase gene in Korean lung cancer patients. Exp Mol Med 1998, 30:192-8.

30. Sugimura H, Wakai K, Genka K, Nagura K, Igarashi H, Nagayama K, Ohkawa A, Baba S, Morris BJ, Tsugane S, Ohno Y, Gao C, Li Z, Takezaki T, Tajima K, Iwamasa T: Association of Ile462Val (Exon 7) polymorphism of cytochrome P450 IA1 with lung cancer in the Asian population: further evidence from a case-control study in Okinawa. Cancer Epidemiol Biomarkers Prev 1998, 7:413-7.

31. Taioli E, Ford J, Trachman J, Li Y, Demopoulos R, Garte S: Lung cancer risk and CYP1A1 genotype in African Americans. Carcinogenesis 1998, 19:813-7.

32. Le Marchand L, Sivaraman L, Pierce L, Seifried A, Lum A, Wilkens LR, Lau AF: Associations of CYP1A1, GSTM1, and CYP2E1 polymorphisms with lung cancer suggest cell type specificities to tobacco carcinogens. Cancer Res 1998, 58:4858-63.

33. Xue KX, Xu Lin, Chen S: Polymorphisms of the CYP1A1 and GSTM1 genes and lung cancer risk in chinese population [in Chinese]. Carcinogenesis Teratogenesis and Mutagenesis 1999, 11:228-230.

34. Hu YL, Zhang Qi: Genetic Polymorphisms of CYP1A1 and Susceptibility of Lung Cancer [in Chinese]. Chin J Med Genet 1999, 16:26-28.

35. Dresler CM, Fratelli C, Babb J, Everley L, Evans AA, Clapper ML: Gender differences in genetic susceptibility for lung cancer. Lung Cancer 2000, 30:153-60.

36. London SJ, Yuan JM, Coetzee GA, Gao YT, Ross RK, Yu MC: CYP1A1 I462V genetic polymorphism and lung cancer risk in a cohort of men in Shanghai, China. Cancer Epidemiol Biomarkers Prev 2000, 9:987-91.

37. Xue KX, Xu Lin, Chen S, Ma GJ, Wu JZ: Polymorphisms of the CYP1A1 and GSTM1 genes and their combined effects on individual susceptibility to lung cancer in a chinese pupulation[in Chinese]. Chin J Med Genet 2001, 18:125-127.

38. Ratnasinghe D, Tangrea JA, Stewart C, Bhat NK, Virtamo J, Albanes D, Taylor PR: Influence of antioxidants and the CYP1A1 isoleucine to valine polymorphism on the smoking-lung cancer association. Anticancer Res 2001, 21:1295-9.

39. Quiñones L, Lucas D, Godoy J, Cáceres D, Berthou F, Varela N, Lee K, Acevedo C, Martínez L, Aguilera AM, Gil L: CYP1A1, CYP2E1 and GSTM1 genetic polymorphisms. The effect of single and combined genotypes on lung cancer susceptibility in Chilean people. Cancer Lett 2001, 174:35-44

40. Song $N$, Tan $W$, Xing D, Lin D: CYP $1 A 1$ polymorphism and risk of lung cancer in relation to tobacco smoking: a case-control study in China. Carcinogenesis 2001, 22:11-6.

41. Chen S, Xue K, Xu L, Ma G, Wu J: Polymorphisms of the CYP1A1 and GSTM1 genes in relation to individual susceptibility to lung carcinoma in Chinese population. Mutat Res 2001, 458:41-47.

42. Zhou X, Shi Y, Zhou Y: The Relationship betweenCYP1A1Genetic Polymorphism and Susceptibility to Lung Cancer [in Chinese]. Chin J Environ Occup Med 2002, 19:355-367.

43. Yin LH, Pu YP, Lin PP: NQO1, CYP1A1, mEH genotype polymorphisms and susceptibility to lung cancer in Nanjing population [in Chinese]. Tumor 2002, 22:14-16.

44. Cai XL, Chen D, Wang BG: Genetic polymorphisms of CYPIAI MsPI and susceptibility of lung Cancer in Guangdong Population[in Chinese]. Jiangsu Prev Med 2003, 14:1-3.

45. Kiyohara C, Wakai K, Mikami H, Sido K, Ando M, Ohno Y: Risk modification by CYP1A1 and GSTM1 polymorphisms in the association of environmental tobacco smoke and lung cancer: a case-control study in Japanese nonsmoking women. Int J Cancer 2003, 107:139-44.

46. Taioli E, Gaspari L, Benhamou S, Boffetta P, Brockmoller J, Butkiewicz D: Polymorphisms in CYP1A1, GSTM1, GSTT1 and lung cancer below the age of 45 years. Int J Epidemiol 2003, 32:60-3. 
47. Wang J, Deng Y, Li L: Association of GSTM1, CYP1A1 and CYP2E1 genetic polymorphisms with susceptibility to lung adenocar- cinoma: a casecontrol study in Chinese population. Cancer Sci 2003, 94:448-452.

48. Dialyna IA, Miyakis S, Georgatou N, Spandidos DA: Genetic polymorphisms of CYP1A1, GSTM1 and GSTT1 genes and lung cancer risk. Oncol Rep 2003, 10:1829-1835.

49. Dong $C T$, Yang $Q$, Wang $M Z$, Dong QN: A study on the relationship between polymorphism of CYP1A1, Lack of GSTM1 and susceptibility to lung cancer [in Chinese]. J Environ Occup Med 2004, 21:440-442.

50. Gu YF, Zhang SC, Lai BT, Wang H, Zhan XP: Relationship between genetic polymorphism of metabolizing enzymes and lung cancer susceptibility [in Chinese]. Chin J Lung Cancer 2004, 7:112-117.

51. Liang GY, Pu YP, Yin LH: Studies of the Genes Related to Lung Cancer Susceptibility in Nanjing Han Population, China [in Chinese]. HEREDITAS 2004, 26:584-588.

52. Chen SD, Ye WY, Chen Q: Relationship between CYP1A1polymorphism, serum selenium and lung cancer[in Chinese]. Chin J Public Health 2004, 20:796-197.

53. Sobti RC, Sharma S, Joshi A, Jindal SK, Janmeja A: Genetic polymorphism of the CYP1A1, CYP2E1, GSTM1 and GSTT1 genes and lung cancer susceptibility in a north indian population. Mol Cell Biochem 2004, 266:1-9.

54. Yang XR, Wacholder S, Xu Z, Dean M, Clark V, Gold B, Brown LM, Stone BJ, Fraumeni JF Jr, Caporaso NE: CYP1A1 and GSTM1 polymorphisms in relation to lung cancer risk in Chinese women. Cancer Lett 2004, 214:197-204.

55. Wrensch MR, Miike R, Sison JD, Kelsey KT, Liu M, McMillan A, Quesenberry C, Wiencke JK: CYP1A1 variants and smoking-related lung cancer in San Francisco Bay area Latinos and African Americans. Int $J$ Cancer 2005, 113:141-7.

56. Ng DP, Tan KW, Zhao B, Seow A: CYP1A1 polymorphisms and risk of lung cancer in non-smoking Chinese women: influence of environmental tobacco smoke exposure and GSTM1/T1 genetic variation. Cancer Causes Control 2005, 16:399-405

57. Larsen JE, Colosimo ML, Yang IA, Bowman R, Zimmerman PV, Fong KM: Risk of non-small cell lung cancer and the cytochrome P4501A1 lle462Val polymorphism. Cancer Causes Control 2005, 16:579-85.

58. Raimondi S, Boffetta P, Anttila S, Bröckmoller J, Butkiewicz D, Cascorbi I: Metabolic gene polymorphisms and lung cancer risk in non-smokers. An update of the GSEC study. Mutat Res 2005, 592:45-57.

59. Sreeja L, Syamala V, Hariharan S, Madhavan J, Devan SC, Ankathil R: Possible risk modification by CYP1A1, GSTM1 and GSTT1 gene polymorphisms in lung cancer susceptibility in a South Indian population. J Hum Genet 2005, 50:618-27.

60. Wenzlaff AS, Cote ML, Bock CH, Land SJ, Santer SK, Schwartz DR, Schwartz AG: CYP1A1 and CYP1B1 polymorphisms and risk of lung cancer among never smokers: a population-based study. Carcinogenesis 2005, 26:2207-12.

61. Adonis M, Martı'nez V, Mari'n P, Gil L: CYP1A1 and GSTM1 genetic polymorphisms in lung cancer populations exposed to arsenic in drinking water. Xenobiotica 2005, 35:519-530.

62. LI DR, Zhou QH, Guo ZL: Relationship between genetic polymorphism of CYP1A1 and lung cancer genetic susceptibility [in Chinese]. Chin J Cancer Prev Treat 2006, 13:1765-1768.

63. Pisani P, Srivatanakul P, Randerson-Moor J, Vipasrinimit S, Lalitwongsa S, Unpunyo P, Bashir S, Bishop DT: GSTM1 and CYP1A1 polymorphisms, tobacco, air pollution, and lung cancer: a study in rural Thailand. Cancer Epidemiol Biomarkers Prev 2006, 15:667-74.

64. Belogubova EV, Ulibina YM, Suvorova IK: Combined CYP1A1/GSTM1 at-risk genotypes are overrepresented in squamous cell lung carcinoma patients but underrepresented in elderly tumor-free subjects. J Cancer Res Clin Oncol 2006, 132:327-331.

65. Jin Y, Yu Z: The effects of CYP1A1 gene polymorphism and $p 16$ gene methylation on the risk of lung cancer [in Chinese]. Acta of Anhui medical University 2007, 42:62-66.

66. Qi XS, Xia Y, Sun QF, Shang B: Association between genetic Polymorphisms ofCYP1A1and Lung Cancer Susceptibility for People Living in High Radon-exposed Area [in Chinese]. Carcinogenesis Teratogenesis and Mutagenesis 2007, 20:11-14.

67. Yang $M$, Choi $Y$, Hwangbo $B$, Lee JS: Combined effects of genetic polymorphisms in six selected genes on lung cancer susceptibility. Lung Cancer 2007, 57:135-42.
68. Cote ML, Wenzlaff AS, Bock CH, Land SJ, Santer SK, Schwartz DR, Schwartz AG: Combinations of cytochrome P-450 genotypes and risk of early-onset lung cancer in Caucasians and African Americans: a population-based study. Lung Cancer 2007, 55:255-62.

69. Xia Y, Sun QF, Shang B: Polymorphisms of the cytochrome P450 and ghtathion s-transferase genes associated with lung cancer susceptibility for the residents in high radon-exposed area [in Chinese]. Chin J Radiol Med Prot 2008, 28:327-332.

70. Yoon KA, Kim JH, Gil HJ, Hwang H, Hwangbo B, Lee JS: CYP1B1, CYP1A1, MPO, and GSTP1 polymorphisms and lung cancer risk in never-smoking Korean women. Lung Cancer 2008, 60:40-6.

71. Gallegos-Arreola MP, Figuera-Villanueva LE, Troyo-Sanroman R, MorgánVillela G, Puebla-Pérez AM, Flores-Marquez MR, Zúniga-González GM: CYP1A1 ${ }^{*} 2 \mathrm{~B}$ and $* 4$ polymorphisms are associated with lung cancer susceptibility in Mexican patients. Int J Biol Markers 2008, 23:24-30.

72. Shah PP, Singh AP, Singh M, Mathur N, Pant MC, Mishra BN, Parmar D: Interaction of cytochrome P4501A1 genotypes with other risk factors and susceptibility to lung cancer. Mutat Res 2008, 639:1-10.

73. Kumar M, Agarwal SK, Goel SK: Lung cancer risk in north Indian population: role of genetic polymorphisms and smoking. Mol Cell Biochem 2009, 322:73-9.

74. Cote ML, Yoo W, Wenzlaff AS, Prysak GM, Santer SK, Claeys GB, Van Dyke AL, Land SJ, Schwartz AG: Tobacco and estrogen metabolic polymorphisms and risk of non-small cell lung cancer in women. Carcinogenesis 2009, 30:626-635.

75. Honma HN, De Capitani EM, Barbeiro Ade S, Costa DB, Morcillo A, Zambon L: Polymorphism of the CYP1A1*2A gene and susceptibility to lung cancer in a Brazilian population. J Bras Pneumol 2009, 35:767-772.

76. Klinchid J, Chewaskulyoung B, Saeteng S, Lertprasertsuke N, Kasinrerk W, Cressey R: Effect of combined genetic polymorphisms on lung cancer risk in northern Thai women. Cancer Genet Cytogenet 2009, 195:143-149.

77. Timofeeva MN, Kropp S, Sauter W, Beckmann L, Rosenberger A, Illig T, Jäger B, Mittelstrass K, Dienemann $\mathrm{H}$, Bartsch $\mathrm{H}$, Bickeböller $\mathrm{H}$, ChangClaude JC, Risch A, Wichmann HE: CYP450 polymorphisms as risk factors for early-onset lung cancer: gender-specific differences. Carcinogenesis 2009, 30:1161-1169.

78. Shaffi SM, Shah MA, Bhat IA, Koul P, Ahmad SN, Siddiqi MA: CYP1A1 polymorphisms and risk of lung cancer in the ethnic Kashmiri population. Asian Pac J Cancer Prev 2009, 10:651-656.

79. Jin $Y, X u$ H, Zhang $C$, Kong $Y$, Hou Y, Xu Y, Xue S: Combined effects of cigarette smoking, gene polymorphisms and methylations of tumor suppressor genes on non small cell lung cancer:a hospital-based casecontrol study in China. BMC Cancer 2010, 10:422.

80. Wright CM, Larsen JE, Colosimo ML, Barr JJ, Chen L, McLachlan RE, Yang IA, Bowman RV, Fong KM: Genetic association study of CYP1A1 polymorphisms identifies risk haplotypes in nonsmall cell lung cancer. Eur Respir J 2010, 35:152-159.

81. Hirschhorn JN, Lohmueller $\mathrm{K}$, Byrne E: A comprehensive reviewof genetic association studies. Genet Med 2002, 4:45-61.

82. Sato S, Nakamura Y, Tsuchiya E: Difference of allelotype between squamous cell carcinoma and adenocarcinoma of the lung. Cancer Res 1994, 54:5652-5.

83. Wydner EL, Hoffman D: Smoking and lung cancer: scientific challenges and opportunities. Cancer Res 1994, 54:5284-95.

84. Engeland A, Andersen A, Haldorsen T, Tretli S: Smoking habits and risk of cancers other than lung cancer: 28 years' follow-up of 26,000 Norwegian men and women. Cancer Causes Control 1996, 7:497-506.

85. Zang EA, Wynder EL: Differences in lung cancer risk between men and women: examination of the evidence. J Natl Cancer Inst 1996, 88(34):183-192.

86. Prescott E, Osler M, Hein HO, Borch-Johnsen K, Lange P, Schnohr P, Vestbo J: Gender and smoking-related risk of lung cancer. The Copenhagen Center for Prospective Population Studies. Epidemiology 1998, 9(1):79-83.

87. Mollerup S, Ryberg D, Hewer A, Phillips DH, Haugen A: Sex differences in lung CYP1A1 expression and DNA adduct levels among lung cancer patients. Cancer Res 1999, 59(14):3317-3320.

88. Siegfried JM: Women and lung cancer: does oestrogen play a role? Lancet Oncol 2001, 2(8):506-513. 
89. Chen Z, Li Z, Niu X, Ye X, Yu Y, Lu S, Chen Z: The effect of CYP1A1 polymorphisms on the risk of lung cancer: a global meta-analysis based on 71 case-control studies. Mutagenesis 2011, 26:437-46.

doi:10.1186/1756-9966-30-99

Cite this article as: Zhan et al:: CYP1A1 Mspl and exon7 gene

polymorphisms and lung cancer risk: An updated meta-analysis and review. Journal of Experimental \& Clinical Cancer Research 2011 30:99.

Submit your next manuscript to BioMed Central and take full advantage of:

- Convenient online submission

- Thorough peer review

- No space constraints or color figure charges

- Immediate publication on acceptance

- Inclusion in PubMed, CAS, Scopus and Google Scholar

- Research which is freely available for redistribution

Submit your manuscript at www.biomedcentral.com/submit
C Biomed Central 